\title{
Bounded rationality can make parking search more efficient: the power of lexicographic heuristics
}

\author{
Merkouris Karaliopoulos \\ Athens University of Economics and Business \\ Department of Informatics \\ 10434 Athens, Greece \\ Konstantinos Katsikopoulos \\ Department of Decision, Analytics, and Risk \\ University of Southampton Business School \\ Southampton SO17 1BJ, UK \\ Lambros Lambrinos \\ Cyprus University of Technology \\ Department of Communication and Internet Studies \\ 3036 Limassol, Cyprus
}

\begin{abstract}
The search for parking space in busy urban districts is one of those routine human activities that are expected to benefit from the widespread adoption of pervasive sensing and radio communication technologies. Proposed parking assistance solutions combine sensors, either as part of fixed infrastructure or onboard vehicles, wireless networking technologies and mobile social applications running on smartphones to collect, share and present to drivers real-time information about parking demand and availability.

One question that arises is how does (and should) the driver actually use such information to take parking decisions, e.g., whether to search for on-street parking space or drive to a parking lot and, in the latter case, which one. The paper is, hence, a performance analysis study that seeks to capture the highly behavioral and heuristic dimension of drivers' decisions and its impact on the efficiency of the parking search process. To this end, and in sharp contrast with the existing literature, we model drivers as agents of bounded rationality and assume that their choices are directed by lexicographic heuristics, an instance of the fast and frugal heuristics developed in behavioral sciences such as psychology and biology. We analyze the performance of the search process under these heuristics and compare it against the predictions of normative game-theoretic models that assume
\end{abstract}


fully rational strategically acting agents. We derive conditions under which the game-theoretic norms turn out to be more pessimistic than the simpler heuristic choice rules and show that these are fulfilled for a broad range of scenarios concerning the fees charged for the parking resources and their distance from the destinations of the drivers' trips. The practical implications of these results for parking assistance solutions are identified and thoroughly discussed.

Keywords: parking search, cognitive decision-making heuristics, congestion games, bounded rationality

\section{Introduction}

The increasing integration of advanced sensing and wireless technologies with urban infrastructures transforms dramatically the way citizens access and interact with them. At the same time, smartphones and other smart mobile devices turn their owners to potential mobile sensing platforms and engage them actively in the generation and distribution of various kinds of information. The two trends combined fuel the smart city concept, whereby fundamental daily activities and operations are carried out more efficiently in favor of individual citizens and the society as a whole Zanella et al. (2014) Greenberg (2015).

Parking space forms an instance of urban resource that is daily accessed and shared by multiple drivers. Often scarce in places such as shopping areas and business districts, it has to be properly managed to avoid congestion effects and the unfavorable consequences of cruising for parking such as the waste of time and fuel and the environmental burden (see, for instance, Shoup (2006)). Parking assistance systems seek to address the parking problem by expanding the reach of pervasive computing within the city roads and turning them to smart spaces. Combining sensors at the parking spots or onboard vehicles with radio communication technologies, and often involving the vehicular network as in Caliskan et al. (2006), they collect and distribute information about parking demand and supply to the vehicular nodes. More recent approaches to the assisted parking search 1 add a social media layer over the vehicular network. The drivers can use a mobile social application running on their smartphones to share their knowledge about parking space with other application users and even handover parking spots to save the overheads of parking search.

\footnotetext{
${ }^{1}$ Examples systems in this context include ParkingCarma (http://www.parkingcarma.com) and ParkShark(http://www.parkshark.mobi/www/)
} 
Despite the amount of work on the proposal and design of parking assistance systems, surprisingly little attention has been paid to their actual performance. Directly relevant to the performance issue is the amount of information that can or should be used when selecting parking resource, e.g., on-street or parking lot(s), or searching for an on-street parking spot. The major assumption in our work is that these decisions involve, in one way or another, humans. The drivers are those who actually make choices when the parking assistance system only provides information about parking resources without making recommendations (although we discuss the implications for systems issuing recommendations in section (7). But even when they are assisted by on-board mounted software agents, the human factor is present either indirectly, through offline configuring the agents with a profile of preferences, or, through its direct participation in the online search and selection processes.

The paper, thus, focuses on the decision-making task and the management of supplied information by the drivers. It seeks to answer how efficient is the parking search process when drivers employ psychologically plausible heuristics to choose between different parking resources. The efficiency of parking search is assessed by how much drivers end up paying in the aggregate to acquire parking spots, including the overhead costs due to needless cruising. The dominant modeling approach in literature is to view drivers as fully rational entities that process all information at hand and act strategically so as to minimize the cost of their search. In behavioral sciences such as economics and psychology, this kind of rationality is called unbounded rationality. In contrast, another kind of rationality is required for problems where there is not adequate time or computational resources in order to obtain all information and find an optimal solution, but nevertheless a good solution must be identified. This is referred to as bounded rationality and Herbert Simon is credited as its father (Simon $(\underline{1955)})$ ).

However, Simon refrained from giving bounded rationality a precise definition and, as a result, multiple viewpoints to it have emerged over time (Rubinstein (1998)Gigerenzer \& Selten (2001) Katsikopoulos (2014)). In the present work, we employ a view drawing on a family of simple mathematical models called fast and frugal heuristics, which are inspired by the actual behavior of people and other animals (Gigerenzer \& Selten (2001)) and instantiate what Simon describes as procedural rationality in Simon (1986). Fast and frugal heuristics include the few pieces of information that people use and also specify the simple ways in which people process this information. Lexicographic heuristics are a specific type of fast and frugal heuristics, where pieces of information 
are inspected sequentially, and decisions are based on the first piece of information which satisfies a pre-determined criterion (such as exceeding a threshold value).

In our paper, which draws on and expands earlier work in Karaliopoulos et al. (2014), we study [4]6 contain the paper's main contributions. Section 4 devises and analyzes the priority heuristic for choosing between the cheap but scarce on-street parking and the spacious yet more expensive parking lot(s). It also compares it with those of the game-theoretic model in Kokolaki et al. (2013). Sections [5] and 6 consider the problem instance with two types of parking lots differentiating in the fees they charge and their distance from the destination of the drivers' trips. Section 5 formulates and analyzes the game that emerges under the assumption of strategic decision-makers, whereas section [6 devises the decision heuristic model drawing on data from field measurements and com- 
pares the two modeling assumptions under different parameter sets. We discuss the implications of our work for actual parking assistance systems in section 7 and argue about its novelty in section

2. while iterating on the existing literature. We conclude our paper in Section 8 .

\section{Related work}

The broader parking space search problem has seen contributions from different scientific disciplines such as economics, transportation, operations research, and computer science. Their majority, however, focus on the performance analysis of given parking systems and relevant strategic planning issues such as where to locate parking resources and how to price them, making assumptions about the way drivers choose parking resources. Fewer address explicitly the drivers' behavior and the decision-making involved in the search process, both descriptively (how do drivers choose parking resources) and normatively (how should they do so to optimize some aspect of the process performance). We summarize them in what follows.

Parking choices as solutions to instances of the optimal stopping problem. The first studies addressing normatively the drivers' choices when searching for parking date back to sixty years ago. Those problem formulations come under the broader family of stopping problems and almost always consider special cases of driving patterns and parking spots' spatial distribution. Indicatively, in MacQueen \& Miller (1960) parking spots are spread randomly with density $\lambda$ over equal-size blocks that are adjacent to the driver's travel destination. Drivers circle through them, crossing the destination every time such a circle is over, and upon encountering a vacancy they decide whether to take it or skip it and seek for a better spot. Whereas Ferguson (Optimal Stopping

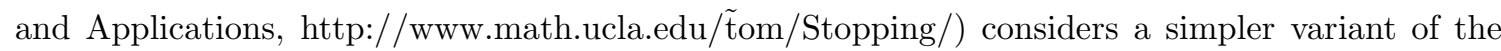
problem, whereby the drivers' destinations lie in the middle of an infinite-length straight line with parking spots that are occupied with probability $p$. In both cases, the optimal prescribed policy is shown to be of the threshold type: drivers should occupy the first vacant spot they hit whenever this lies within some distance $r=f(\lambda)$, resp. $f(p)$, from their destination and continue their search otherwise. Much more recently in Chen et al. (2016), a stopping problem formulation is derived for the same setting (infinite-length straight line with parking spots) when drivers are given information about the occupation probability of each spot along the line.

Parking choices as individual decision-making instances. More formal probabilistic logit and probit models (and variants thereof) for the parking choices drivers make are assumed and cali- 
brated in Van Der Goot (1982), Lambe (1996), and Hunt \& Teply (1993). In Van Der Goot (1982), a logit chance model is trained out of a survey in the central area of the city of Haarlem, in Netherlands. The utilities assigned to the parking alternatives are weighted sums of mixed linear and non-linear functions of the walking time, charges, occupation rates, possible parking-time restrictions, and accessibility factorsrelated to different parking alternatives. Yet, the reported accuracy of the model (over the same data that were used for training it) is quite low: in most cases, the percentage of instances that the alternative indicated by the model as the most probable alternative and the actual choice coincide varies from $21.4 \%$ to $64.5 \%$. A similar modeling approach within a more elaborate setting with 55 parking lots and 13 different trip destinations in the Central Business District(CBD) of Vancouver, Canada, is followed in Lambe (1996). Both a multinomial probit and a logit model are tried to predict the parking choices of drivers. Contrary to Van Der Goot (1982), the utilities assigned to the parking alternatives weigh additively their driving (from the trips' origins) and walking (from the trips' destinations) distances, and the fee they charge. On a similar note, a nested logit model with two levels is proposed in Hunt \& Teply (1993). The first modeling level concerns the type of parking resource (on-street parking vs. parking lot and, cases that parking is arranged by the employer) and the second one captures the choice of spot within the particular type, e.g., a curbside spot or a specific lot. As a result, three different utility functions are formulated, one at the first level and two at the second level. All of them are weighted additive functions of typical attributes such as the charged fee, their walking distance from the trip destination), but also atypical ones such as the type and condition of parking surface in each alternative. The model is trained through data from 344 interviews in the CBD of Edmonton, Canada. Finally, a similar model for parking choice is formulated in Florian \& Los (1980). The aim is to predict the occupation of a number of park 'n ride lots co-located with stations of a commuters' rail transit line in Philadelphia, US. They use two different datasets, obtained in different but time-adjacent periods, for training their model and testing its prediction accuracy, respectively, which lies in the order of $5 \%$ for all but a couple of parking lots.

Two remarks are worth making regarding the aforementioned thread of logit (and multinomial probit) models. First, they are calibrated/trained against aggregate data averaged over all drivers. Namely, the utility functions of all users (or partitions of them grouped according to the type or timing of their trips) weigh the different decision attributes with the same weights-only in Lambe (1996) some differentiation is allowed through the introduction of a random error term giving rise to 
the proposed multinomial logit choice model. Secondly, they are all instances of individual decisionparking lots. The authors find the equilibria of the resulting congestion game and the resulting inefficiency (a.k.a Price of Anarchy) that results from their individual decisions. They also carry out a sensitivity analysis on how these are affected by the adopted pricing policies. 
A conceptually similar model, with two clusters/lots of parking spots (a nearby one of finitecapacity and higher fee vs. a remote but cheap and larger one) is treated by Qian \& Rajagopal (2014). They focus on the morning commuters' setting, where the parking demand is distributed over a specific time interval and take a dynamic programming approach to control the price of the near lot so that the total time spent on the parking search (including the walking and driving components plus the time-equivalent of the parking fee) is minimized. The utilities assigned to the two lots by each user vary along the weight each assigns to the fee charged by the lot (the reciprocal of what they call Value of Time). Inputs to the price determination process are the distribution of parking demand over the different time periods, collected sensor data about the current occupancies of the lot, and the utilities assigned to the two lots by individual users. The price of the lot is then monotonically increased over time to ensure that the portion of users accumulating to the lot does not completely saturate its capacity. The work in Qian \& Rajagopal (2014) is part of a larger thread focusing on the interaction of road traffic congestion and parking demand during commuting hours. In Qian et al. (2012), the standard morning commute model of Vickrey is enriched with two clusters of parking lots and drivers choose one strategically iterating on the overall cost of each choice, which includes both the parking fees and the inconvenience due to driving, walking and early/late arrival times to their office. This model is then used to derive guidelines as to how the location, fees and capacities of the two parking resources affect the aggregate cost the users incur. On the downside, all users are assumed homogeneous, i.e., they assess all incurred delays and costs the same way. On the contrary, classes of users, differentiating with respect to their trip purpose and parking duration are considered in Lam et al. (2006). The authors apply the theory of variational inequalities over the transportation network that involves the road network and the parking facilities. For given origin-destination demands, the model can compute the variation of traffic flows over time across the network; yet its complexity grows fast with the networks size limiting its practical applications. Finally, more macroscopic models of the same theme (i.e., interaction of road traffic with parking demand) are proposed in Cao \& Menendez (2015) and Boyles et al. (2015). These models can be used to derive measures of road traffic and average walking/driving times with Bovles et al. (2015) and without Cao \& Menendez (2015) modeling the underlying road network.

In our work, we emphasize on the decision-making aspects underlying the choice of parking resource. As in Du \& Gong (2016) Kokolaki et al. (2013), Qian \& Rajagopal (2014) and Qian et al. (2012), drivers choose among different types of parking resources, namely on-street parking spots 
and parking lots, and their decisions jointly determine how many parking spots are occupied and how many end up paying the undesirable cruising cost. However, we radically depart from existing literature in the way we model the drivers' decision-making process. Contrary to all aforementioned studies, we view drivers as boundedly rational agents, whose decisions are driven by cognitive heuristics, i.e., heuristics pointing to intuitive cognitive processes, rather than probabilistic or strategic models driven by some notion of utility function maximization. Whereas bounded rationality has been explored as a modeling framework for drivers' behavior in the route choice problem (see Katsikopoulos et al. (2002) and Di \& Liu (2016) for an exhaustive survey of relevant models), to the best of our knowledge and understanding, there are only two pieces of marginally relevant experimental work on the parking search problem from Hester et al. (2002) and Hutchinson et al. (2012), respectively. Hester et al. (2002) found that when parking lots are arranged in a straight line, parking behavior in a driving simulator is described well by the following lexicographic heuristic: consider parking lots one at a time-starting from the one closest to the destination -and choose to park in the first lot where the number of available spots exceeds a threshold. Hutchinson et al. (2012) run simulations to study the parking search outcome in a straight line parking network, when all drivers activate a simple parking search heuristic called "the fixed distance heuristic": park in the first vacant spot as soon as you lie within some threshold range from your trip's destination, which is reminiscent of the policy prescribed in MacQueen \& Miller (1960)).

\section{Background}

A typical dilemma faced by drivers when approaching their destinations in busy urban areas is whether to invest time and effort in searching for cheaper on-street parking space or drive directly towards one of the more expensive parking lots. The first option involves the risk of failing to find a vacant spot and eventually paying, in addition to the parking fees, an excess cost due to cruising in terms of fuel consumption and wasted time.

\subsection{The parking spot selection game}

In Kokolaki et al. (2013), this dilemma is formulated as a resource selection problem. On-street parking space and parking lot(s) are two discrete types of parking resources with per time unit $\operatorname{costs} c_{1}$ and $c_{2}$, respectively, with $c_{1}<c_{2}$. An additional excess cost $c_{\text {exc }}$ becomes relevant when vehicles end up in a parking lot after failing to seize an on-street parking spot. The vehicular 

the available information to them. The outcome of their actions and the respective payoffs depend on the decisions of all nodes so that their interaction is formulated as a game, the parking spot selection game:

Definition 1. The Parking Spot Selection Game is a tuple $\Gamma_{1}(N)=\left(\mathcal{N}, \mathcal{R},\left(A_{i}\right)_{i \in \mathcal{N}},\left(w_{j}\right), j \in\{1,2\}\right)$, 240 where:

- $\mathcal{N}=\{1, \ldots, N\}, N>1$ is the set of drivers searching for parking space,

- $\mathcal{R}=\mathcal{R}_{1} \cup \mathcal{R}_{2}$ is the set of parking spots; $\mathcal{R}_{1}$ is the set of on-street spots, with $R=\left|\mathcal{R}_{1}\right| \geq 1$; $\mathcal{R}_{2}$ is the set of spots in the parking lot(s), with $\left|\mathcal{R}_{2}\right| \geq N$,

- $A_{i}=\{1,2\}$ is the action set for each driver $i \in \mathcal{N}$, action "1" denoting search for on-street 245 parking space and "פ" driving directly to a parking lot,

- $w_{1}(\cdot)$ and $w_{2}(\cdot)$ are the cost functions of the two actions, respectively.

where

$$
w_{1}(n)=\min (1, R / n) c_{1}+\max (0,1-R / n)\left(c_{2}+c_{e x c}\right)
$$

and $w_{2}(n)=c_{2}, n \in[1, N]$.

It is shown in Kokolaki et al. (2013) that at the Nash Equilibrium (NE) states of the game, whether pure or mixed, the vehicular nodes tend to over-compete for the scarce on-street parking space. Namely, the vehicles, $N_{c m p}^{N E}$, that choose to compete for on-street parking space outnumber its supply, $R$, so that some of them end up in a parking lot only after incurring the excess cruising cost, $c_{e x c}$. More formally, the game $\Gamma_{1}(N)$ has:

- a single pure strategy $\mathrm{NE}, N_{c m p}^{N E}=N$, or equivalently a single symmetric mixed NE with $p_{c m p}^{N E}=1$, if $N \leq N_{0}$

- $\left(\begin{array}{c}N \\ \left\lfloor N_{0}\right.\end{array}\right)$ pure NE with $N_{c m p}^{N E}=\left\lfloor N_{0}\right\rfloor$, if $N>N_{0}$ and $N_{0}$ is not an integer,

- $\left(\begin{array}{c}N \\ N_{0}\end{array}\right)$ pure NE with $N_{c m p}^{N E}=N_{0}$ and $\left(\begin{array}{c}N \\ N_{0}-1\end{array}\right)$ pure NE with $N_{c m p}^{N E}=N_{0}-1$, if $N>N_{0}$ and $N_{0}$ is an integer,

- one symmetric mixed NE with $p_{c m p}^{N E}=N_{0} / N$, if $N>N_{0}$ 
where

$$
N_{0}=\frac{c_{2}+c_{e x c}-c_{1}}{c_{e x c}} R
$$

In either case, and given that $N_{c m p}^{N E}=N \cdot p_{c m p}^{N E}$, the aggregate cost (fees+excess cost) paid by the drivers equals

$$
C_{a g g}^{N E}=\min \left(R, N_{c m p}^{N E}\right) c_{1}+\max \left(0, N_{c m p}^{N E}-R\right)\left(c_{2}+c_{e x c}\right)+\left(N-N_{c m p}^{N E}\right) c_{2}
$$

The aggregate cost paid by the drivers is the measure of the parking search process efficiency

throughout the paper under all devised models. For the game $\Gamma_{1}(N)$, the ratio of this cost, $C_{a g g}^{N E}$, over the one achieved by an optimal centralized assignment of parking space, $C_{\text {opt }}=R c_{1}+(N-R) c_{2}$, is a typical measure of the inefficiency that the lack of central coordination induces. The worstcase value of this ratio over all possible game instances determines the Price of Anarchy (PoA) (see Koutsoupias \& Papadimitriou (2009)) of the game $\Gamma_{1}(N)$. Its sensitivity to the different game parameters is exhaustively studied in Kokolaki et al. (2013).

\subsection{Fast and frugal decision heuristics}

The NE is a solution concept with strong implications for the capabilities of decision-makers: they are agents with adequate processing capacity to analyze the available information and assess the alternatives presented to them. Most importantly, they can also analyze the possible strategies of other agents and identify the equilibrium choice, from which they have no reason to unilaterally deviate.

In this paper we place emphasis instead on a breed of heuristics developed in behavioral sciences such as psychology and biology, called fast and frugal heuristics (Gigerenzer et al. (2011)). According to the definition given in Katsikopoulos (2011), fast and frugal heuristics are models for making decisions, that: (i) rely heavily on core human capacities (such as memory recognition and recall); (ii) do not necessarily use all available information, and process the information they use by simple computations (such as using only one piece of information); (iii) are easy to understand, apply, and explain. Hence, it is no surprise that they have been proposed as relevant to a broad range of human decision-making tasks such as medical diagnosis in Marewski \& Gigerenzer (2012) and consumer decisions in Kurz-Milcke \& Gigerenzer (2007).

Lexicographic heuristics represent probably the most important and recognized category of these heuristics, not least because specific properties they possess contrast them strongly with the full rationality prescriptions. Hence, lexicographic heuristics do not induce tradeoffs between the decision 


\begin{tabular}{|c|c|}
\hline Symbol & Context \\
\hline$n_{1}$ & number of drivers choosing the on-street parking space \\
\hline$n_{2}$ & number of drivers choosing the remote parking lot \\
\hline$R\left(R_{1}\right)$ & number of on-street parking spots \\
\hline$R_{2}$ & capacity of remote parking lot \\
\hline$c_{1}$ & per unit time cost of on-street parking spots \\
\hline \multirow[t]{2}{*}{$c_{2}$} & cost of parking lot(s) in the 1st parking search problem \\
\hline & cost of the remote parking lot in the 2 nd parking search problem \\
\hline$c_{e x c}$ & excess cruising cost \\
\hline$c_{3}$ & cost of the expensive parking lot in the 2 nd parking search problem \\
\hline$\Gamma_{1}(N)$ & strategic game corresponding to the 1st parking search problem \\
\hline$\Gamma_{2}(N)$ & strategic game corresponding to the 2 nd parking search problem \\
\hline$f_{r}(r)$ & function relating distance to monetary cost \\
\hline$N_{c m p}^{N E}$ & number of drivers choosing on-street parking spots at the equilibrium of $\Gamma_{1}(N)$ \\
\hline \multirow[t]{2}{*}{$N_{c m p}^{P H}, N_{P L}^{P H}$} & number of drivers choosing on-street parking spots (resp. parking lot) \\
\hline & under the prioriy heuristic in the 1st parking search problem \\
\hline \multirow[t]{2}{*}{$N_{i}^{N E}$} & number of drivers choosing on-street parking spots $(i=1)$, the remote parking lot $(i=2)$ \\
\hline & and the expensive parking lot $(\mathrm{i}=3)$ in $\Gamma_{2}(N)$ \\
\hline \multirow[t]{2}{*}{$N_{i}^{P H}$} & number of drivers choosing on-street parking spots $(i=1)$, the remote parking lot $(i=2)$ \\
\hline & and the expensive parking lot $(\mathrm{i}=3)$ under the lexicographic heuristic \\
\hline \multirow[t]{2}{*}{$t h r_{c}$} & threshold value for the ratio of the minimum costs' difference over the worst-case cost \\
\hline & $\left(c_{2}-c_{1}\right) /\left(c_{2}+c_{e x c}\right)$, under the two alternatives in the 1st parking search problem \\
\hline$F_{t h r_{c}}()$ & cumulative distribution function of $t h r_{c}$ \\
\hline \multirow[t]{2}{*}{$t h r_{p}$} & threshold value for the difference of the probabilities of incurring minimum costs \\
\hline & under the two alternatives in the the 1st parking search problem \\
\hline$F_{t h r_{p}}()$ & cumulative distribution of $t h r_{p}$ \\
\hline$r_{d c}$ & decision certainty ratio, equal to $\frac{c_{2}-c_{1}}{c_{e x c}}$ \\
\hline$F_{t h r_{f}}()$ & $\begin{array}{l}\text { cumulative distribution function of the acceptability threshold value for the parking fee } \\
\text { in the } 2 \text { nd parking problem instance }\end{array}$ \\
\hline$F_{t h r_{d}}()$ & $\begin{array}{l}\text { cumulative distribution function of the acceptability threshold value for the distance of the parking resource } \\
\text { from the trip's destination in the } 2 \text { nd parking problem instance }\end{array}$ \\
\hline
\end{tabular}

Table 1: Table of symbols. 
attributes, i.e., they are non-compensatory; they do not necessarily process the full information at hand or the full set of attributes in the decision process; and do not assign to each alternative an overall score (a.k.a utility) that serves as basis for comparison.

For example, consider the priority heuristic for choices among risky alternatives (interchangeably called gambles in the relevant literature), as introduced in Brandstatter et al. (2006). Let us say we want to choose one of $\mathrm{X}=\left(x_{\min }, p ; x_{\max }, 1-p\right)$ and $\mathrm{Y}=\left(y_{\min }, q ; y_{\max }, 1-q\right)$, where for $\mathrm{X}$ the numerical outcome $x_{\min }$ is obtained with probability $\mathrm{p}$ and $x_{\max }$ is obtained with 1 - $\mathrm{p}$ and $0<x_{\min }<x_{\max }$ (and analogously for $\mathrm{Y}$ ).

If $\left|x_{\min }-y_{\min }\right|>t h r_{1} \cdot \max \left\{x_{\max }, y_{\max }\right\}$, no other attributes are inspected and the gamble with the higher value on its minimum outcome is chosen. Otherwise, if $|p-q|>t h r_{2} 2$, a decision is made in favor of the gamble with the lower probability of minimum outcome without considering attributes beyond the second. In the opposite case, the gamble with the higher maximum outcome is chosen (and if the maximum outcomes are equal, a choice is made randomly).

Another instance of lexicographic heuristics, which models human choice between multiple multiattribute choices under certainty, is the LEX heuristic (Payne et al. (1993)). In LEX, the decision makers first rank the attributes in order of decreasing importance $\left(x_{1}, x_{2}, \ldots\right)$, which is highly subjective and depends on their individual preferences. They then inspect the value of all alternatives on the first attribute $x_{1}$. If all alternatives tie on $x_{1}$, this attribute is eliminated. If a single alternative outperforms the rest on $x_{1}$, this alternative is chosen. Otherwise, all alternatives with a value on $x_{1}$ worse than the best one are eliminated and the process is repeated by using the second attribute $x_{2}$. The process continues in this way until an alternative is chosen. If more than one alternatives are left after all attributes are inspected, then a choice is made randomly.

Closely related to LEX is the deterministic elimination by aspects (DEBA) heuristic in Hogarth \& Karelaia (2005). DEBA introduces acceptability threshold values for each attribute, which determine whether the specific attribute value for an alternative is acceptable or not. As a result, each alternative can described by a series of ones (attribute value is acceptable) and zeros (attribute value is nonacceptable). Otherwise, the selection process under DEBA proceeds as under LEX. Namely, the attributes are ordered in decreasing order of importance $\left(x_{1}, x_{2}, \ldots\right)$ by the decision makers, in line

\footnotetext{
${ }^{2}$ The value 0.1 is used for both thresholds $t h r_{1}$ and $t h r_{2}$ in Brandstatter et al. (2006) as a global constant across all humans.
} 
with their individual preferences. Alternatives are inspected on the first attribute $x_{1}$ and those featuring non-acceptable values (a zero) are eliminated. If a single alternative remains, it is chosen. If none remains, an alternative is chosen at random. If there are more than one alternatives with an ace in this attribute, the process is repeated by parsing the second attribute $x_{2}$. The process continues in this way until an alternative is chosen. If more than one alternatives are left after all attributes are inspected, then a choice is made randomly.

The research on the performance of lexicographic heuristics has been progressing along two main, rather independent, threads drawing on both computer simulations and mathematical analysis. The first one focuses on the heuristics' accuracy to explain and, more importantly, predict human decisions as these are logged in a variety of datasets from the fields of business, medicine and psychology. Two major stylized facts have emerged in this respect (Katsikopoulos (2011)). First, they lead to choices which, in terms of quality, perform comparably to, and sometimes even better than, more complex choice models such as linear regression, classification and regression trees, neural and Bayesian networks; in particular, simple heuristics are less prone to overfitting and often have higher performance in out-of-sample prediction. Secondly, each one of the heuristics or benchmarks outperforms the other under certain conditions. The priority heuristic, for instance, predicts very well which economic risks people prefer, sometimes outperforming standard behavioral economics models such as prospect theory Katsikopoulos \& Gigerenzer (2008).

The second research thread on these heuristics explores their capacity to explain major empirical phenomena that violate the normative decision-making prescriptions and have repeatedly reported in various experimental or real-life settings. For example, it has been analytically shown in Katsikopoulos \& Gigerenzer (2008) that the priority heuristic predicts a host of such phenomena in risky choice such as violations of the common consequence and common ratio axioms as well as the four-fold pattern of risk attitudes, as described in Kahneman (2011).

In what follows, we consider an implementation of the priority heuristic when drivers choose between the two parking space resources considering the fees charged for the parking resources in combination with the risk of not finding a vacant spot. Later in section [6 we let decisions also take account of the distances of the parking spots from the drivers' destinations. 


\section{The priority heuristic for selecting parking resource}

Intuitively, when making their decisions, the drivers weigh the higher fee of the parking lot option against the risk of failing to seize an on-street parking spot and having to pay this fee plus the excess cruising cost. The increased cost of the parking lot is essentially a premium the drivers are called to pay for overcoming the risk of searching for on-street parking. Generally, the premium each driver is willing to pay varies and depends on her financial status, the type/purpose of her trip (e.g., leisure vs. business) and her assessment of the magnitude of the risk. Hence, for some drivers even a small premium in the fee the parking lot operator charges serves as a deterrent. On the contrary, other drivers may find the higher parking lot fees an acceptable price to pay for the increased convenience and time savings lots offer.

\subsection{Formulation of the heuristic}

We devise an implementation of the priority heuristic for the parking choice problem drawing on its generic description in Section 3. Since the problem setting involves losses (costs) rather than gains, the heuristic prescribes that drivers consider first the minimum cost related to each parking alternative; then the probabilities that these costs emerge out of their choices; and, finally, if no decision is made by that time, the maximum costs they may incur when choosing the one or the other alternative. More specifically, invoking the notation in Section 3.2 the on-street parking space can be viewed as the risky gamble $X=\left(-c_{1}, \min (1, R / N) ;-\left(c_{2}+c_{e x c}\right), 1-\min (1, R / N)\right)$, whereas the option of the parking lot as the fixed-outcome gamble $Y=-c_{2}$ with probability 1 . Then

- (step 1) drivers decide to search for on-street parking space if the minimum possible costs of the two alternatives, $c_{1}$ and $c_{2}$ respectively, differ by more than a percentage $t h r_{c}$ of the worst-case cost they may incur as a result of their selection (i.e., $\left.c_{2}+c_{e x c}\right)$.

- (step 2) In the opposite case, i.e., $u=\frac{c_{2}-c_{1}}{c_{2}+c_{e x c}} \leq t h r_{c}$, they postpone their decision until after considering the probabilities of minimum costs: if their difference exceeds a threshold $t h r_{p}$, they head for a parking lot.

- (step 3) Otherwise, when $1-\min (1, R / N)<t h r_{p}$ so that neither the second criterion helps them reach a clear decision, they compare the maximum possible costs, $c_{2}+c_{\text {exc }}$ and $c_{2}$, and decide in favor of the parking lot option. 


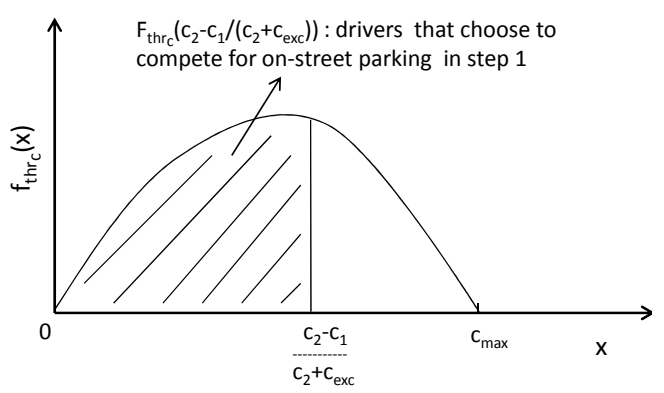

a. Parking fees/cost

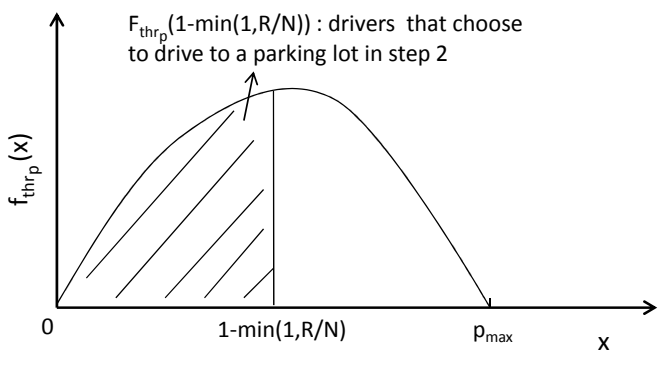

b. Parking demand

Figure 1: Probability distributions for the sensitivity of drivers to the minimum parking cost (left) and the respective probabilities (right)

As mentioned in Section 3.2, the original generic description of the priority heuristic in Brandstatter et al. (2006) recommends a single driver-agnostic constant (i.e., 0.1) for both threshold parameters, $t h r_{c}$ and $t h r_{p}$. We have argued instead that these values typically vary across drivers reflecting differences in their financial status, the reason of their trip (business or leisure) and their individual preferences. This heterogeneity of drivers is modeled statistically through two probability distribution functions, $f_{t h r_{c}}$ and $f_{t h r_{p}}$ respectively.

Letting $F_{t h r_{c}}$ and $F_{t h r_{p}}$ be the respective cumulative distribution functions, the number of drivers who compete for on-street parking spots equals (ref. Fig. 11)

$$
N_{c m p}^{P H}=N \cdot F_{t h r_{c}}\left(\frac{c_{2}-c_{1}}{c_{2}+c_{e x c}}\right)
$$

whereas the drivers that opt for the parking lot alternative are

$$
N_{P L}^{P H}=N \cdot\left(1-F_{t h r_{c}}\left(\frac{c_{2}-c_{1}}{c_{2}+c_{e x c}}\right)\right)
$$

Note that the partitioning of drivers to the two groups is independent of the distribution $f_{t h r_{p}}$.

Drivers who do not decide to compete for on-street parking space in step 1 will end up in a parking lot, either in step 2 or in the last step. The distribution $f_{t h r_{p}}$ only determines the split between the drivers deciding in favor of a parking lot in step 2 and those doing so in the last step.

In analogy with (3), the aggregate cost (fees+excess cost) paid by the drivers equals

$$
C_{a g g}^{P H}=\min \left(R, N_{c m p}^{P H}\right) c_{1}+\max \left(0, N_{c m p}^{P H}-R\right)\left(c_{2}+c_{e x c}\right)+\left(N-N_{c m p}^{P H}\right) c_{2}
$$


Table 2: Conditions for more efficient parking resource selection under the priority heuristic.

\begin{tabular}{|c|cc|}
\hline Relation of A, B & Corresponding $r_{d c}$ range \\
\hline \hline$A>B$ & $r_{d c}<B$ & $(\mathrm{C} 1)$ \\
& $r_{d c} \geq A$ & $(\mathrm{C} 2)$ \\
& $\max \left(B, \frac{N \cdot F_{t h r_{c}}\left(\frac{c_{2}-c_{1}}{c_{2}+c_{e x c}}\right)-R}{R}\right) \leq r_{d c}<A$ & $(\mathrm{C} 3)$ \\
& & \\
& $r_{d c} \geq B$ & $(\mathrm{C} 4)$ \\
& $r_{d c}<A$ & $(\mathrm{C} 5)$ \\
& $A \leq r_{d c}<\min \left(B, \frac{N-R}{N \cdot F_{t h r_{c}}\left(\frac{c_{2}-c_{1}}{c_{2}+c_{e x c}}\right)-R}\right)$ & $(\mathrm{C} 6)$ \\
\hline \hline \multicolumn{2}{|c|}{$B=\frac{N-R}{R}, \quad B=\left(1+\frac{c_{2}}{c_{e x c}}\right) \cdot F_{t h r_{c}}^{-1}(R / N)$} \\
\hline
\end{tabular}

\subsection{Analytical comparison with the game model $\Gamma_{1}(N)$}

It is then convenient to analyze the efficiency of the parking search process under the game and the priority heuristic models as a function of the decision certainty ratio $r_{d c}=\left(c_{2}-c_{1}\right) / c_{e x c}$. This ratio reflects the overall attractiveness of the on-street parking alternative: it grows as the parking fee difference increases or the expected cruising cost due to the risk related to competing for on-street parking, declines. Theorem 1 summarizes the comparison of the two decision-making models.

Theorem 1. The parking search process when drivers' choices are driven by the priority heuristic is more efficient than when they act as fully rational strategic agents under the conditions listed in Table 2 .

The proof is provided in Appendix A.

Corollary 1. The parking search process when drivers' choices are driven by the priority heuristic is coherently more efficient than when they act as fully rational strategic agents as long as either:

$$
\frac{N \cdot F_{t h r_{c}}\left(\frac{c_{2}-c_{1}}{c_{2}+c_{e x c}}\right)-R}{R}<\left(1+\frac{c_{2}}{c_{e x c}}\right) \cdot F_{t h r_{c}}^{-1}(R / N)<\frac{N-R}{R}
$$

or

$$
\frac{N-R}{R}<\left(1+\frac{c_{2}}{c_{e x c}}\right) \cdot F_{t h r_{c}}^{-1}(R / N)<\frac{N-R}{N \cdot F_{t h r_{c}}\left(\frac{c_{2}-c_{1}}{c_{2}+c_{e x c}}\right)-R}
$$



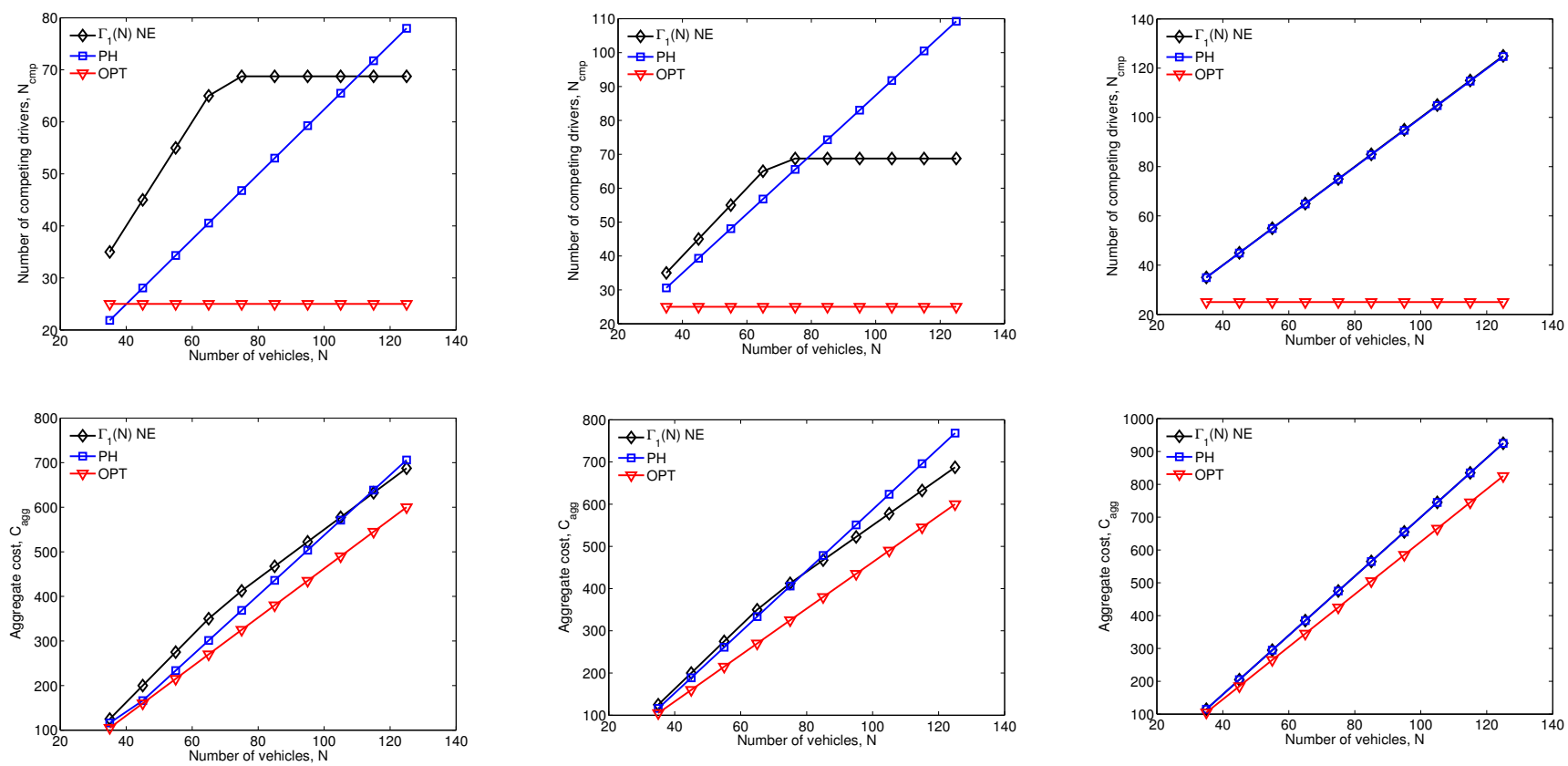

a. $c_{1}=2, c_{2}=5.5, c_{e x c}=2, c_{\max }=0.8$

b. $c_{1}=2, c_{2}=5.5, c_{e x c}=2, c_{\max }=0.6$

c. $c_{1}=1, c_{2}=8, c_{e x c}=1, c_{\max }=0.8$

Figure 2: Number of drivers competing for on-street parking and aggregate cost as a function of the parking demand: $R=25$.

Essentially, Table 2 sketches the conditions under which the activation of the priority heuristic induces less over-competition for the on-street parking spots than when drivers are treated as fully rational agents who decide strategically during their parking search.

What are plausible choices for the distribution $f_{t h r_{c}}$ ? One such choice would be a parabolic function of the type $f_{t h r_{c}}(x)=\alpha x^{2}+\beta x+\gamma$ over $\left[0, c_{\max }\right]$ implying that more drivers take the risk to compete for medium values of the $u$ ratio rather than at very low or high fee differences. Imposing $\int_{0}^{c_{\max }} f_{t h r_{c}}(x)=1$ and $f_{t h r_{c}}(0)=f_{t h r_{c}}\left(c_{\max }\right)=0$, we get

$$
f_{t h r_{c}}(x)= \begin{cases}\frac{6 x}{c_{\max }^{2}}\left(1-\frac{x}{c_{\max }}\right) & \text { if } x \in\left[0, c_{\max }\right] \\ 0 & \text { otherwise }\end{cases}
$$

\subsection{Numerical results}

We draw on the analysis of Section 4.2 to demonstrate the operational dynamics of the parking search process under the two decision-making models as a function of the charged fees $c_{1}, c_{2}$, the 
drivers' sensitivity to their difference $\left(c_{\max }\right)$, and the cruising cost $c_{e x c}$. As a side comparison reference, we consider the achievable assignment of parking space by a centralized system with perfect information about parking space demand and availability.

The first recurring, as well as intuitive, trend in all plots of Fig. 2 is the dominance of the centralized assignment scheme (noted as OPT in subsequent plots). The scheme sets a benchmark by achieving optimal aggregate parking cost. The game-theoretic equilibria of $\Gamma_{1}(N)$, on the other hand, result in a cost that exceeds the optimal by a ratio that varies depending on the scenario.

Far more interesting is to track how the cost induced by bounded-rational decisions compares with that corresponding to the game equilibria and when is the related inefficiency due to overcompetition for the on-street parking space amplified/attenuated. In Fig. $2 \mathrm{a}\left(r_{d c}=1.75\right)$ the game model prescribes that $\min \left(N_{0}=68.75, N\right)$ vehicles should be competing for on-street parking space. The priority heuristic, on the other hand, requires that a fixed $62 \%$ of the population would do so, resulting in under-utilization of on-street parking space for $N<40$. The aggregate cost remains consistently lower under the priority heuristic even when demand exceeds supply by a fraction of four $(N / R>4)$. The heuristic model generates higher aggregate cost only for $N>110$, where the condition (C3) of Table 2 is violated (for $N=110, A=3.6, B=0.95, N \cdot F_{t h r_{c}}\left(\frac{c_{2}-c_{1}}{c_{2}+c_{e x c}}\right)-R$ ) $/ R=1.74$ ).

As drivers become more sensitive to the charged fee difference (Fig. $2 \mathrm{~b}, c_{\max }=0.6$ ), the use of the priority heuristic directs more of them to compete for on-street parking space. The aggregate paid cost exceeds that at the NE of the strategic game faster but not before the demand/supply ratio $N / R$ exceeds 3 (for $\left.N=80, A=2.4, B=0.837, N \cdot F_{t h r_{c}}\left(\frac{c_{2}-c_{1}}{c_{2}+c_{e x c}}\right)-R\right) / R=1.796$ ).

The two decision models give identical predictions when the fee difference $c_{2}-c_{1}$ : a) renders the parking lot option prohibitively expensive for all drivers deciding heuristically; b) does not let the expected cost of competition for on-street parking space in (1), exceed the parking lot fee for any value of (realistic) parking demand. This is the case plotted in Fig. 2 ;.

A final remark worth making is that this analysis is not very informative as to how individual drivers fare under the two decision-making assumptions. Depending on which specific $\Gamma_{1}(N)$ game equilibrium is realized out of the $\mathrm{N}$ over $N_{c m p}^{N E}$ possible ones, there are drivers who are better off when activating the priority heuristic and others who are worse off. The former set includes: a) drivers who seize an on-street parking spot under the heuristic but do not do so under strategic decision-making; b) drivers who directly drive to the parking lot under the heuristic, whereas they decide to compete for on-street parking space without success, under strategic decision-making. 
The analysis informs us how many drivers end up in each of the two parking alternatives and how many pay the excess cruising cost but not how individual drivers fare in the two cases.

\section{Two parking lots - fully rational decision makers}

The assumption in the decision problem of Section 3.1 is that the parking lots do not differentiate with respect to their location and fees. Equivalently, all parking lots are located within approximately the same area (e.g., business district) and they charge the same fee for their parking space so that drivers do not have strong preference for the one or the other.

In what follows, we relax these two assumptions, introducing differentiation regarding both the fees charged by the different parking resources and their distances from the trip destination. We consider two parking lots (could be lot classes) as alternatives to on-street parking. The first one is co-located with the drivers' common travel destination (distance $r_{3}=0$ ) and charges a fee $c_{3}$ per time unit. The second one is further away at distance $r_{2}>0$ and charges a fee $c_{2}$, with $c_{1}<c_{2}<c_{3}$. On a more technical note, these assumptions yield a multi-attribute choice problem with three alternatives. As before, we first formulate a game model for it, which becomes relevant under the highly normative assumption that that the vehicular nodes decide and act strategically and fully rationally; this is covered in the current section. We then tailor the simpler LEX and DEBA heuristics (ref. Section 3) to the problem and compare the solutions they induce against the NE of the game model in Section 6 .

The game model that captures the strategic interactions of drivers under full rationality stands in direct analogy with $\Gamma_{1}(N)$, as defined in Section 3.1 .

Definition 2. The Extended Parking Spot Selection Game is a tuple $\Gamma_{2}(N)=\left(\mathcal{N}, \mathcal{R},\left(A_{i}\right)_{i \in \mathcal{N}},\left(w_{j}\right), j \in\right.$ $\{1,2,3\})$, where:

- $\mathcal{N}=\{1, \ldots, N\}, N>1$ is the set of drivers searching for parking space,

- $\mathcal{R}=\mathcal{R}_{1} \cup \mathcal{R}_{2} \cup \mathcal{R}_{3}$ is the set of parking spots; $\mathcal{R}_{1}$ is the set of on-street spots, with $\left|\mathcal{R}_{1}\right|:=$ $R_{1} \geq 1 ; \mathcal{R}_{2}$ is the set of spots in the more distant yet cheaper parking lot, with $\left|\mathcal{R}_{2}\right|:=R_{2} \geq 1$ and $R_{1}+R_{2}<N$; and $\mathcal{R}_{3}$ is the set of parking spots in the spacious but expensive lot that lies next to the travel destination, with $\left|\mathcal{R}_{3}\right|:=R_{3}>N$.

- $A_{i}=\{1,2,3\}$ is the action set for each driver $i \in \mathcal{N}$, action "1" denoting search for on-street parking space, "2" driving directly to the cheaper and "3" to the more expensive parking lot. 
With respect to the original game $\Gamma_{1}(N)$, cost functions have now to account for the non-monetary cost related to the distance of the three alternatives and the effort related to driving and walking from/to them. If $f_{r}(\cdot)$ is a function that monetizes this cost, it holds that $f_{r}\left(r_{2}\right)>f_{r}\left(r_{1}\right)>f_{r}\left(r_{3}\right)=$ 0 .Then, assuming that drivers who fail to seize an on-street parking spot will first seek for parking space in the distant cheaper lot and only if this does not work out for them, they will resort to the expensive lot with guaranteed parking space, $w_{1}\left(n_{1}, n_{2}\right)$ is given by 3

$$
\begin{aligned}
w_{1}\left(n_{1}, n_{2}\right) & =\min \left(1, \frac{R_{1}}{n_{1}}\right)\left(c_{1}+f_{r}\left(r_{1}\right)\right)+\left\lceil 1-\frac{R_{1}}{n_{1}}\right\rceil^{+}\left(\min \left(1, \frac{\left\lceil R_{2}-n_{2}\right\rceil^{+}}{\left\lceil n_{1}-R_{1}\right\rceil^{+}+\left\lceil n_{2}-R_{2}\right\rceil^{+}}\right)\left(c_{2}+c_{e x c}+f_{r}\left(r_{2}\right)\right)\right. \\
& \left.+\max \left(0,1-\frac{\left\lceil R_{2}-n_{2}\right\rceil^{+}}{\left\lceil n_{1}-R_{1}\right\rceil^{+}+\left\lceil n_{2}-R_{2}\right\rceil^{+}}\right)\left(c_{3}+c_{\text {exc }}\right)\right)
\end{aligned}
$$

Likewise, for the cost of deciding to opt for the cheaper parking lot, we have

$$
w_{2}\left(n_{1}, n_{2}\right)=\min \left(1, \frac{R_{2}}{n_{2}}\right)\left(c_{2}+f_{r}\left(r_{2}\right)\right)+\left\lceil 1-R_{2} / n_{2}\right\rceil^{+}\left(c_{3}+c_{e x c}\right)
$$

and, finally, $w_{3}\left(n_{1}, n_{2}\right)=c_{3}$, where $\lceil x\rceil^{+}=\max (x, 0)$ and $\left(n_{1}, n_{2}\right)$ describes the state of the system, i.e., the numbers of vehicular nodes that decide to compete for on-street parking capacity and drive towards the cheaper distant parking lot, respectively (so that $N-n_{1}-n_{2}$ is the number of vehicular nodes that select the more expensive parking lot).

In principle, $f_{r}(\cdot)$ is driver-specific; extra driving and walking effort is not equally annoying to all drivers. Yet for computational tractability purposes, we restrict ourselves to symmetric users, assuming that $f_{r}(\cdot)$ has similar semantics for all users and adopting the functional form in Shoup (1999) for it. In all cases considered in the paper, it holds that $f_{r}\left(r_{2}\right)+c_{2}<c_{3}$, i.e., the distance

\footnotetext{
${ }^{3}$ It could be argued that users who choose to compete for on-street parking and end up in the remote parking lot should see smaller cruising cost than those who end up in the expensive parking lot, after getting all the way to the remote lot and back. Likewise, the argument could continue, the cruising cost of drivers who originally choose the remote parking lot but end up in the expensive parking lot should lie in-between the two earlier costs. This way, we would have three different "versions" of this cost. We have chosen instead to use the variable $c_{e x c}$ to invariably denote the cruising cost, which incurs whenever a driver fails parking in his/her original parking resource choice. This way we avoid getting into discussions of the kind "Is this cost proportional to the distance or subject to another rule?" and save added complexity that does not add any worthwhile insights to the problem.
} 
of the remote parking lot does not alone compensate for its reduced fee. Defining:

$$
\begin{aligned}
N_{12} & =\left(1+\frac{c_{2}+f_{r}\left(r_{2}\right)-\left(c_{1}+f_{r}\left(r_{1}\right)\right)}{c_{\text {exc }}}\right) R_{1} \\
N_{13} & =\left(1+\frac{c_{3}-\left(c_{1}+f_{r}\left(r_{1}\right)\right)}{c_{\text {exc }}}\right) R_{1} \\
N_{23} & =\left(1+\frac{c_{3}-\left(c_{2}+f_{r}\left(r_{2}\right)\right)}{c_{\text {exc }}}\right) R_{2} \quad \text { and } \\
N_{123} & =N_{13}+N_{23}
\end{aligned}
$$

we can show that

Theorem 2. The pure $N E$ of the $\Gamma_{2}(N)$ game are listed in Table [3, when $N_{12} \leq R_{1}+R_{2}$ and in Table 4, when $N_{12}>R_{1}+R_{2}$.

The proof is provided in Appendix B.

Proposition 1. For any value of $N>R_{1}$, the $N E$ of the game $\Gamma_{2}(N)$ induce over-competition for the on-street parking capacity.

The proof is given in Appendix C.

\section{Two parking lots - bounded-rational decision makers}

The game-theoretic model for the drivers' decisions among the three alternatives has normative rather than descriptive value. It implies that, besides acquiring and sharing information about the demand and supply of parking resources and exhaustively processing it, vehicular nodes also need to "monetize" the cost related to the distance of parking resources from the drivers' destination. In what follows, we fundamentally shift our hypothesis for the way drivers make their decisions. We assume that behind these decisions lie heuristics reflecting specific cognitive processes. As in Section 4, our focus in on lexicographic heuristics, in particular the LEX and DEBA heuristics described in Section 3.2

Intuitively, drivers are highly heterogeneous regarding both the actual heuristics they activate and the way they configure them (e.g., the order in which the decision criteria are parsed). Ideally, we would need real data that could serve as training datasets for the assumed heuristic models. For a given user, such datasets could be historical data about his/her past choices in similar selection setups. We could then apply standard machine learning techniques to infer which heuristic each 
Table 3: Pure NE of $\Gamma_{2}(N)$ for different values of $N$, case: $N_{12} \leq R_{1}+R_{2}$

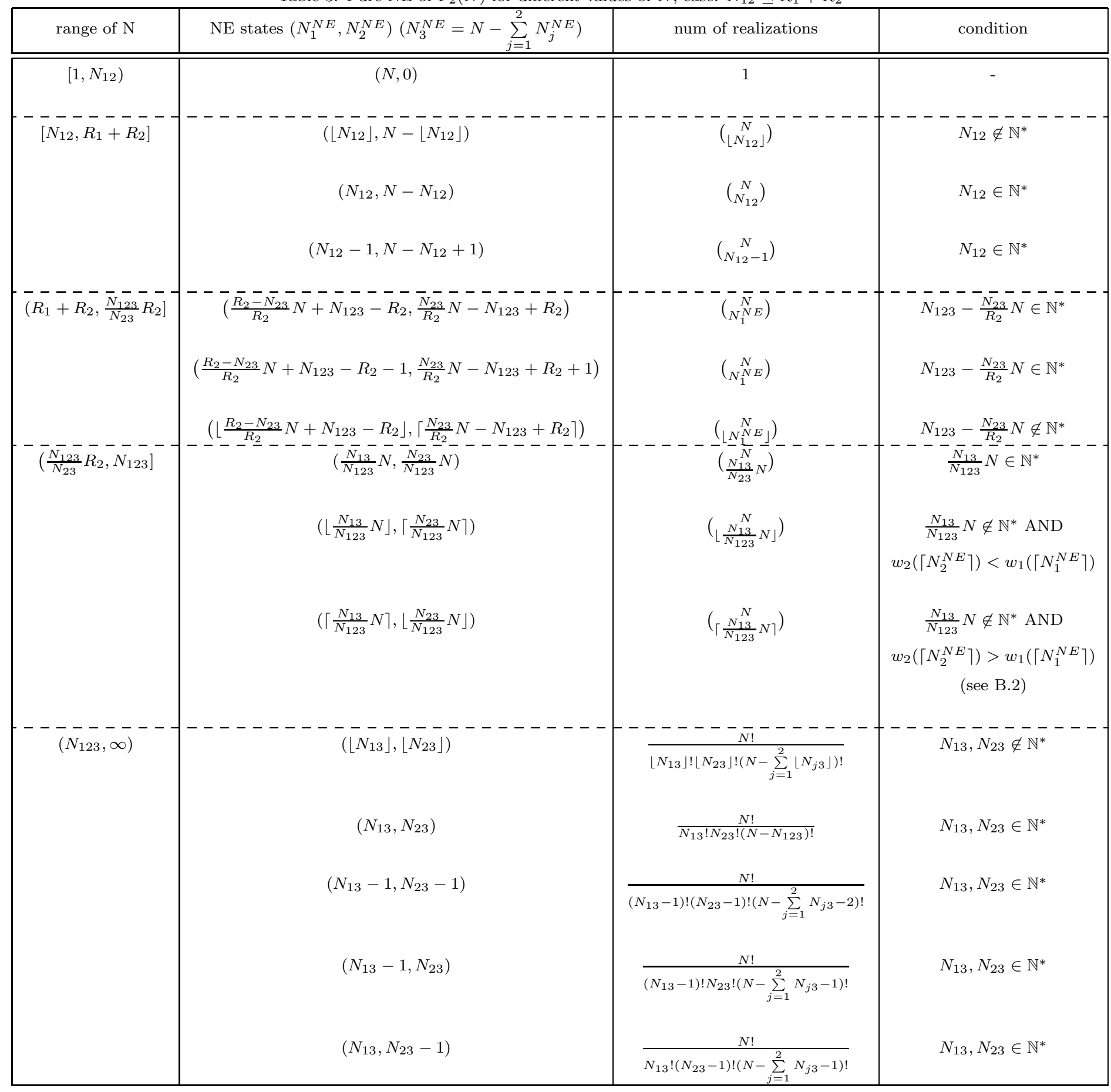


Table 4: Pure NE of $\Gamma_{2}(N)$ for different values of $N$, case: $N_{12} \geq R_{1}+R_{2}$

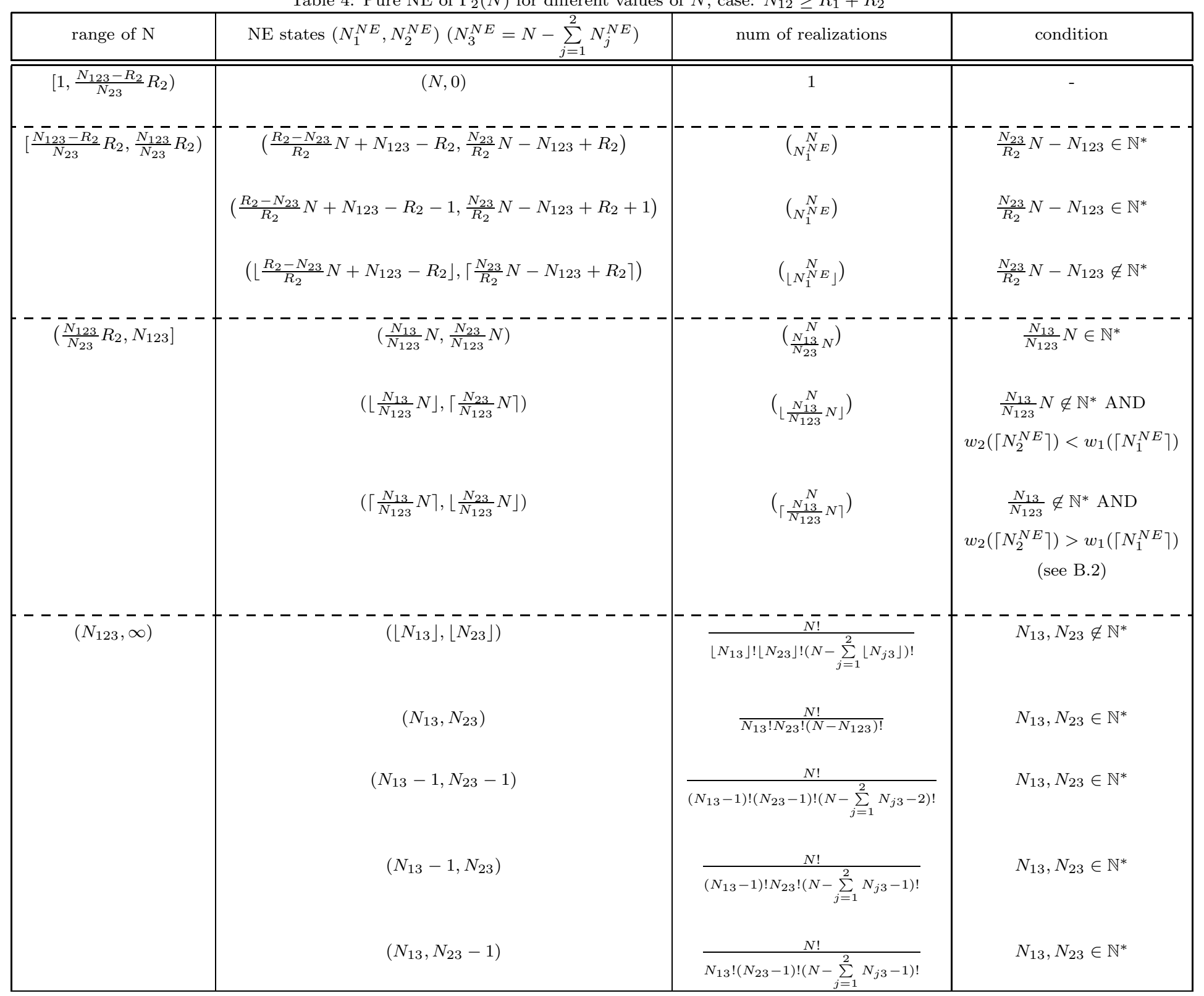


driver most probably activates and estimate its parameters, such as how does $\mathrm{s}($ he) prioritize the decision criteria (e.g., distance, parking fee), and which are the relevant acceptability thresholds. We are not aware of such datasets that could serve the model training purpose (we comment further on this in section 7). Rather than synthesizing arbitrary random scenarios about the drivers' heterogeneity, we preferred to make the most out of survey data about drivers' parking habits. In what follows, we describe these survey data and explain how we use them to deduce a more realistic scenario about the way drivers could activate the LEX and DEBA heuristics (rank of decision criteria, thresholds) and their resulting choices.

\subsection{Survey data and the inference of drivers' heuristics}

The survey was conducted in June 2012 and involved approximately 1120 drivers. The majority (around 800) of the drivers were asked to complete the paper-based questionnaire on-the-spot just after they had parked at a 300-space parking lot in the center of Nicosia, Cyprus, whereas the remaining answers were gathered from an online questionnaire (posing exactly the same questions as the paper version). The survey took place on different days and times of the week in order to acquire data from drivers who came to the city center for business purposes as well as leisure (e.g., shopping, eating out etc.) but also cover peak and off-peak periods of demand for parking. The full questionnaire consisted of 42 questions seeking to fully capture the subjects' driving and parking experience and habits. The core of the questionnaire aimed at identifying the drivers' requirements from public parking infrastructures, the problems they currently face in their use, and how technological solutions can offer service enhancements.

The replies of drivers about their parking resource preferences are summarized in Figure 3 and reflect high heterogeneity in their decision criteria. First, as shown in Figure $3 \mathrm{a}$, the drivers differentiate with respect to their preferred parking resource type, with approximately $14 \%$ of them parking only in lots, $5 \%$ parking always on-street, and the rest using both types of parking resources. Secondly, they prioritize different criteria for choosing among alternatives of a given parking resource type. Notably, Fig. 3b suggests that the relative weight of the parking fee and distance in the selection of resource varies with the parking resource type preferences. For instance, out of the drivers who stated that they park exclusively in parking lots, around $15 \%$ choose among parking lots based on the fees charged by them, almost $37 \%$ consider only their distances from their trip's destination, whereas the remaining drivers take into account both criteria. On the contrary, 


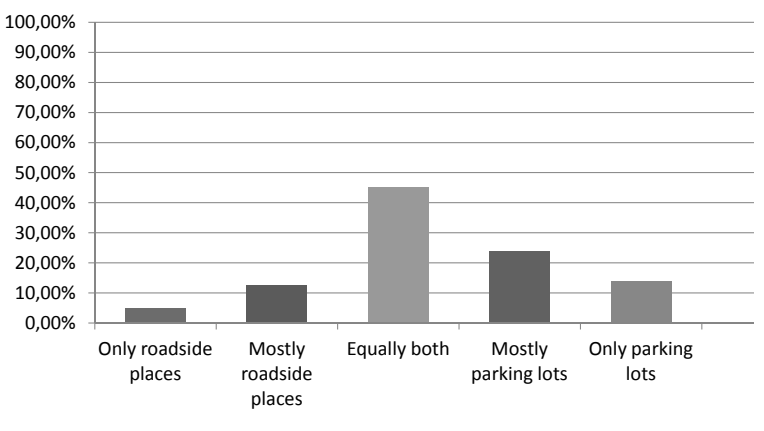

(a) Parking resource used

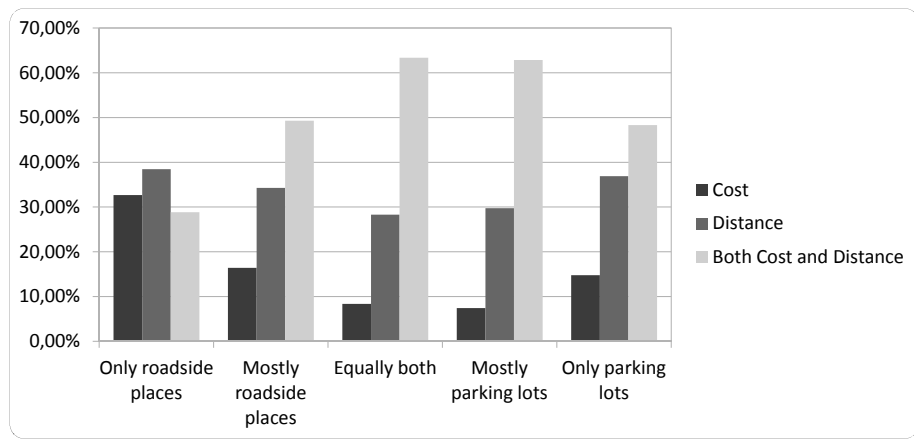

(b) Criteria for selecting parking space for each of the five categories in (a)

Figure 3: Survey outcomes regarding the parking preferences of 1120 drivers in Nicosia, Cyprus.

the respective percentages for those who park exclusively on-street, are $32.7 \%, 38.45 \%$, and $28.85 \%$, respectively.

We carried out a correlation analysis for the drivers' parking preferences. More formally, the preferred parking resource type and the weight of parking fee and distance in the selection of parking resource can be viewed as two categorical variables $p$ and $q$. The first one categorizes the drivers into five groups, $p_{i}, i \in[1,5]$ being the percentage volume of drivers in each one so that $\sum_{i} p_{i}=1$. The second variable further partitions each group $i \in[1,5]$ into three subgroups $q_{i j}, j \in[1,3]$, namely ("exclusively cost", "exclusively distance", "both"), with $\sum_{j} q_{i j}=1$. For instance, $q_{41}$ denotes the portion of drivers out of those "mostly parking their cars in lots", who consider only the charged fees for choosing a lot. Carrying out the chi-square independence test for the categorical variables $p$ and $q$, the null hypothesis that $p$ and $q$ are not inter-related (hence, independent) is emphatically rejected even at $1 \%$ level of significance $\left(p-\right.$ value $\left.=8.3 \cdot 10^{-9}\right)$.

Relating lexicographic heuristics to each of the possible fifteen groups of drivers determined by the two categorical variables $p$ and $q$ is rather intuitive and shown in Table 5 . Therefore, drivers using consistently one type of parking resource, either on-street spots or parking lots, and relying their decision exclusively on the parking fee or distance, seem to decide in line with the LEX heuristic. These drivers order the parking resource type as the first (binary) decision attribute, and the parking fee or distance as second, respectively. Likewise, drivers who use both parking resource types and depend their decision exclusively on the parking fee (distance), could be view as LEX decision makers that consider a single decision attribute, i.e., fee (resp. distance), resembling 
Table 5: Mapping of lexicographic heuristics to the drivers' choices. In parentheses is shown the order of inspected attributes for each category: $t \rightarrow$ parking type, $f \rightarrow$ fee, $d \rightarrow$ distance.

\begin{tabular}{|c|c|c|c|c|c|}
\hline & only on-street & mostly on-street & equally both & mostly lots & only lots \\
\hline \hline fee & $\operatorname{LEX}(\mathrm{t}, \mathrm{f}, \mathrm{d})$ & $\operatorname{LEX}(\mathrm{f}, \mathrm{d})$ & $\operatorname{LEX}(\mathrm{f}, \mathrm{d})$ & $\operatorname{LEX}(\mathrm{f}, \mathrm{d}))$ & $\operatorname{LEX}(\mathrm{t}, \mathrm{f}, \mathrm{d})$ \\
distance & $\operatorname{LEX}(\mathrm{t}, \mathrm{d}, \mathrm{f})$ & $\operatorname{LEX}(\mathrm{d}, \mathrm{f})$ & $\operatorname{LEX}(\mathrm{d}, \mathrm{f})$ & $\operatorname{LEX}(\mathrm{d}, \mathrm{f})$ & $\operatorname{LEX}(\mathrm{t}, \mathrm{d}, \mathrm{f})$ \\
both fee and distance & $\operatorname{DEBA}(\mathrm{t}, \mathrm{f}, \mathrm{d})$ & $\operatorname{DEBA}(\mathrm{f}, \mathrm{d})$ & $\operatorname{DEBA}(\mathrm{f}, \mathrm{d})$ & $\operatorname{DEBA}(\mathrm{d}, \mathrm{f})$ & $\operatorname{DEBA}(\mathrm{t}, \mathrm{d}, \mathrm{f})$ \\
\hline
\end{tabular}

the Take The Best (TTB) heuristic used for inference tasks in Gigerenzer \& Goldstein (1996). On the other hand, drivers considering both the fee and distance factors in their choices, can be better modeled as DEBA decision-makers. Inherent to DEBA are satisfiability thresholds for each decision attribute turning the continuous values of the fee and distance attributes to zeros and ones. These driver-specific thresholds together with the actual decision setting (fees and distances of lots and on-street spots) determine which is the decisive attribute in each case.

\subsection{Analysis of the system performance under the heuristic decision model}

Given a population $N$ of drivers seeking for parking space, the expected number of drivers who search for on-street parking can be written as

$$
N_{1}^{H}=N \cdot\left(p_{1}+\sum_{j=2}^{4} p_{j}\left(q_{j 1}+x_{j} \cdot q_{j 3}\right)\right)
$$

and includes three groups: (a) those parking exclusively on-street $\left(p_{1}\right)$; (b) drivers who decide according to the charged fee only out of those generally using both roadside spots and lots, $\left(q_{j 1}\right.$, $j \in\{2,3,4\}$ ); and, finally, (c) part $\left(x_{j}\right)$ of the drivers in the same three groups who consider both the charged fee and distance when making their decisions $\left(q_{j 3}\right)$.

Likewise, the expected number of drivers who will end up in the distant but cheap parking lot can be written as

$$
N_{2}^{H}=N \cdot\left[p_{5}\left(q_{51}+y_{5} \cdot q_{53}\right)+\sum_{j=2}^{4} p_{j} \cdot q_{j 3} \cdot y_{j}\right]
$$

to include (a) drivers who use parking lots exclusively and decide on the basis of the charged fee only $\left(q_{51}\right)$; (b) part $\left(y_{5}\right)$ of the drivers who use parking lots exclusively and state that they take into account for their 
decisions both the charged fee and the cost $\left(q_{53}\right)$; and (c) part $\left(y_{j}, j \in\{2,3,4\}\right)$ of those drivers who use both types of parking resources $\left(q_{j 3}\right)$ and inspect both distance and fees when deciding for parking space.

Finally, the drivers who end up in the expensive but destination-adjacent lot are

$$
N_{3}^{H}=N \cdot\left[p_{5}\left(q_{52}+q_{53} \cdot z_{5}\right)+\sum_{j=2}^{4} p_{j}\left(q_{j 2}+q_{j 3} \cdot z_{j}\right)\right]
$$

This group includes (a) drivers using exclusively parking lots and selecting among them according to their distances from the trip destination $\left(p_{5} \cdot q_{52}\right)$; (b) a portion $z_{5}$ of drivers using exclusively parking lots and considering both distance and fees for selecting one out of more alternatives; and, (c) part of the drivers who tend to park in both parking resource types and base their choices on both the distance and fee charged by the alternatives.

Intuitively, it holds that

$$
x_{j}+y_{j}+z_{j}=1 \quad j \in\{2,3,4\} \text { and } y_{5}+z_{5}=1
$$

\subsubsection{Determining the $x, y$, and $z$ vectors}

The drivers who do not express a strict preference for one of the two types of parking resources, accounting for $81 \%$ of the total population according to Fig. 3a, will decide on a case-by-case basis considering multiple criteria: the fee charged by each alternative, the availability of spots and possible competition for them, and the distance of each resource from their trip destination. The importance of the criteria varies across drivers, each one exhibiting different sensitivity to the values assumed by them. We capture this heterogeneity through specifying statistical distributions for the value of each criterion that is considered acceptable by them, i.e., the threshold values of fee and distance, beyond which a particular alternative is rendered prohibitive.

Let $F_{t h r_{f}}(x), x \in\left[c_{\min }, c_{\max }\right]$ be the cumulative distribution function of the maximum acceptable parking fee threshold across drivers; and $F_{t h r_{d}}(x), x \in\left[0, d_{\max }\right]$ the respective function for the maximum acceptable distance of spots from the trip's destination. For instance, $F_{t h r_{d}}(0)$ would correspond to the portion of the driver population who would be reluctant to park to any place that is not destination-adjacent. Ideally, these distributions could emerge after monitoring the drivers' behavior over some interval of time and learning their preferences.

With these distributions at hand, the portion of drivers who choose to compete for the on-street 


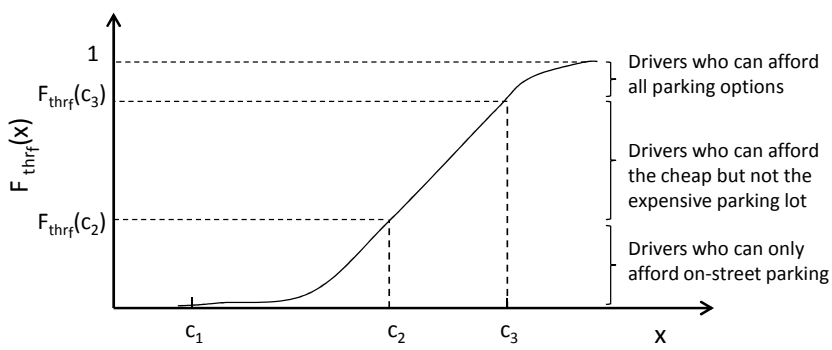

a. Fees

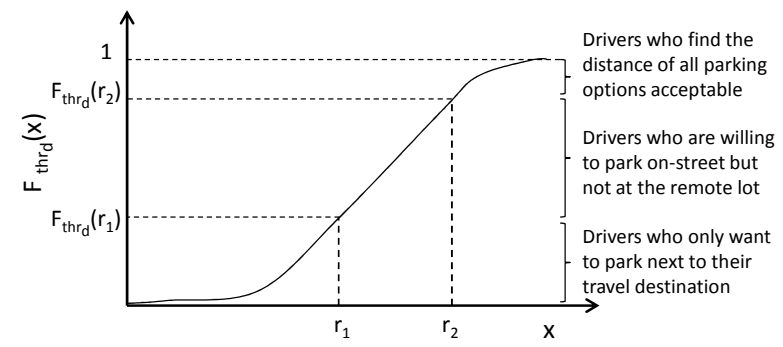

b. Distance

Figure 4: Cumulative probability distributions for the fees drivers are willing to pay (left) and distance they are willing to traverse (right) for parking

parking spots can be expressed as:

$$
\begin{aligned}
x_{2}=x_{3} & =F_{t h r_{c}}\left(c_{2}\right)+\left(F_{t h r_{f}}\left(c_{3}\right)-F_{t h r_{f}}\left(c_{2}\right)\right) \cdot\left(\left(F_{t h r_{d}}\left(r_{2}\right)+\left(1-F_{t h r_{d}}\left(r_{2}\right)\right) / 2\right)\right) \\
& \left.+\left(1-F_{t h r_{f}}\left(c_{3}\right)\right) \cdot\left(\left(F_{t h r_{d}}\left(r_{2}\right)-F_{t h r_{d}}\left(r_{1}\right)\right) / 2+\left(1-F_{t h r_{d}}\left(r_{2}\right)\right) / 3\right)\right)
\end{aligned}
$$

In words, this subset of drivers includes: (a) those who are not willing to pay even the fee of the cheaper parking lot; (b) part of those who fee-wise also consider the cheap parking lot option but eventually head for on-street parking because they deem the distance of the lot prohibitive or randomly select between two otherwise equivalent options; and (c) those willing to pay even the price of the expensive parking lot (see Fig. 情) and distance-wise view either one or both parking lots as acceptable options, as Fig. 4b suggest:4].

On the other hand, $x_{4}$ relates to drivers first inspecting the distance of the parking resource alternative. Hence,

$$
\begin{aligned}
x_{4} & =\left(F_{t h r_{d}}\left(r_{2}\right)-F_{t h r_{d}}\left(r_{1}\right)\right) \cdot\left(F_{t h r_{f}}\left(c_{3}\right)+\left(1-F_{t h r_{f}}\left(c_{3}\right)\right) / 2\right) \\
& +\left(1-F_{t h r_{d}}\left(r_{2}\right)\right) \cdot\left(F_{t h r_{f}}\left(c_{2}\right)+\left(F_{t h r_{f}}\left(c_{3}\right)-F_{t h r_{f}}\left(c_{2}\right)\right) / 2+\left(1-F_{t h r_{f}}\left(c_{3}\right)\right) / 3\right)
\end{aligned}
$$

\footnotetext{
${ }^{4}$ Recall that the DEBA model prescribes that whenever two or more alternatives pass the thresholds of all attributes, the user draws randomly one of those. This is how the factors $1 / 2$ and $1 / 3$ emerge in the second and third summands.
} 


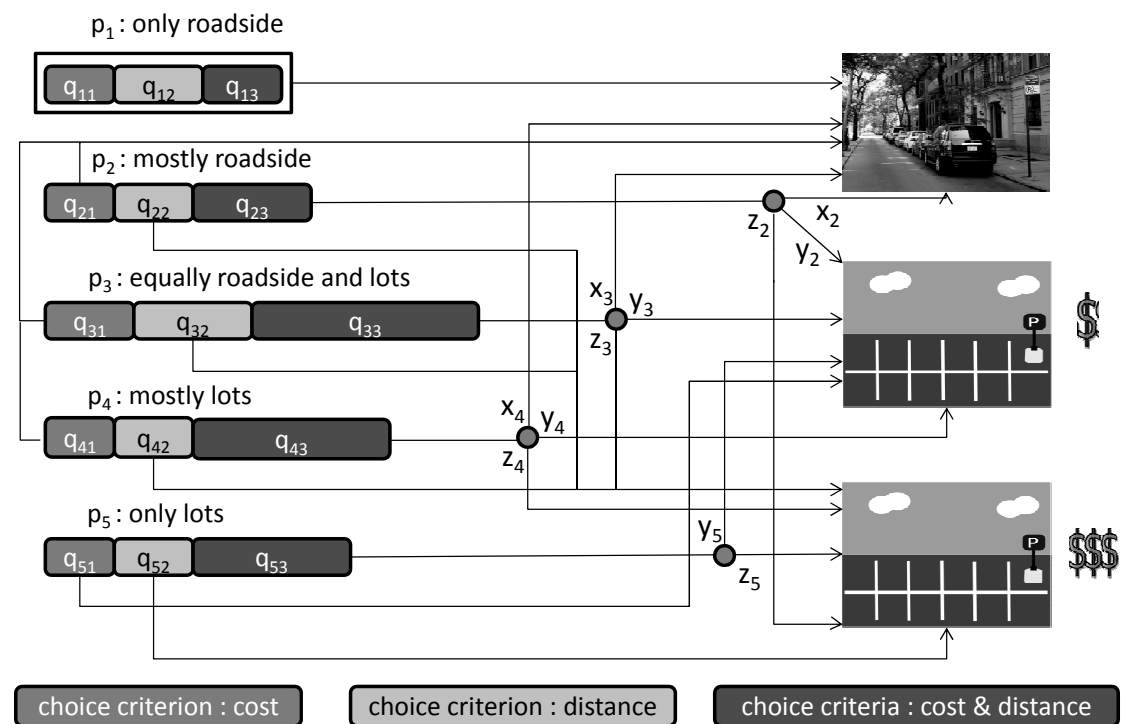

Figure 5: From survey data to drivers' choices. On the left is shown the two-level grouping of drivers out of their survey responses, as shown in Fig. 3 The numbers of drivers that choose each one of the three parking resources on the right, are composed by different groups. The factors $x_{i}, i \in\{2,3,4\}, y_{j}, j \in\{2,3,4,5\}, z_{k}, k \in\{2,3,4,5\}$ represent the percentages of drivers considering both the fees and distances of the parking resource that end up choosing the on-street parking, the cheap distant lot, and the destination-colocated expensive one, respectively. 
Likewise, we can express the portion of drivers who choose the cheaper but distant parking lot as:

$$
y_{2}=y_{3}=\left(F_{t h r_{f}}\left(c_{3}\right)-F_{t h r_{f}}\left(c_{2}\right)\right) \cdot\left(1-F_{t h r_{d}}\left(r_{2}\right)\right) / 2+\left(1-F_{t h r_{f}}\left(c_{3}\right)\right) \cdot\left(1-F_{t h r_{d}}\left(r_{2}\right)\right) / 3
$$

namely, $y_{2}$ and $y_{3}$ include those who can afford the cheap parking lot, or even the more expensive one, and are not deterred from its distance. On the other hand,

$$
y_{4}=\left(1-F_{t h r_{d}}\left(r_{2}\right)\right) \cdot\left(\left(F_{t h r_{f}}\left(c_{3}\right)-F_{t h r_{f}}\left(c_{2}\right)\right) / 2+\left(1-F_{t h r_{f}}\left(c_{3}\right)\right) / 3\right)
$$

hence, to end up in the cheap parking lot when considering first the distance attribute, one would need to view its distance as acceptable and not rendering it at disadvantage with respect to the other two alternatives.

Similarly,

$$
y_{5}=\left(1-F_{t h r_{d}}\left(r_{2}\right)\right) \cdot\left(F_{t h r_{f}}\left(c_{3}\right)+\left(1-F_{t h r_{f}}\left(c_{3}\right)\right) / 2\right)
$$

since $y_{5}$ partitions a group of drivers always opting for parking lots $(i . e$., on-street parking is not an option).

Finally, the portions of drivers who choose to drive to the more expensive destination-adjacent lot among those considering all three options and inspect the charged fee first, are given by

$$
z_{2}=z_{3}=\left(1-F_{t h r_{f}}\left(c_{3}\right)\right) \cdot\left(F_{t h r_{d}}\left(r_{1}\right)+\left(F_{t h r_{d}}\left(r_{2}\right)-F_{t h r_{d}}\left(r_{1}\right)\right) / 2+\left(1-F_{t h r_{d}}\left(r_{2}\right)\right) / 3\right)
$$

and basically comprise those who are willing to pay its fee and appreciate, though to different extents, its advantage over the other two alternatives in terms of distance from the trip destination. On the other hand, when the distance attribute is inspected first, the portion of drivers who end up in the expensive lot is given by

$$
z_{4}=F_{t h r_{d}}\left(r_{1}\right)+\left(F_{t h r_{d}}\left(r_{2}\right)-F_{t h r_{d}}\left(r_{1}\right)\right) \cdot\left(1-F_{t h r_{f}}\left(c_{3}\right)\right) / 2+\left(1-F_{t h r_{d}}\left(r_{2}\right)\right) \cdot\left(1-F_{t h r_{f}}\left(c_{3}\right)\right) / 3
$$

when drivers are a priori open to all three options, and

$$
z_{5}=F_{t h r_{d}}\left(r_{2}\right)+\left(1-F_{t h r_{d}}\left(r_{2}\right)\right) \cdot\left(1-F_{t h r_{f}}\left(c_{3}\right)\right) / 2
$$

for drivers exclusively considering parking lots. 
Table 6: Distributions of threshold values for acceptable charged fees and parking resource distance (in minutes walking) across the drivers' population.

\begin{tabular}{|c|c|c|}
\hline Choice parameter & Distribution & range \\
\hline \hline max acceptable fee & & \\
$F_{t h r_{f}}(x)$ & $\frac{-6 \cdot\left(\frac{x^{3}}{3}-\left(c_{\max }+c_{\min }\right) \cdot \frac{x^{2}}{2}+c_{\min } \cdot c_{\max } \cdot x-\frac{c_{\min }^{3}}{3}+\left(c_{\min }+c_{\max }\right) \cdot \frac{c_{\min }^{2}}{2}-c_{\min }^{2} \cdot c_{\max }\right)}{\left(c_{\max }-c_{\min }\right)^{3}}$ & {$\left[c_{\min }, c_{\max }\right]$} \\
$\max$ distance & & \\
$F_{t h r_{d}}(x)$ & $\frac{6 \cdot\left(x^{2} / 2-x^{3} / 3 / r_{\max }\right)}{r_{\max }^{2}}$ & {$\left[0, r_{\max }\right]$} \\
\hline
\end{tabular}

However the decision is made, strategically or in line with the lexicographic heuristics, the aggregate cost that drivers will have to pay is a function of their partitioning to the three groups

$$
\begin{aligned}
& \left\{N_{1}^{a}, N_{2}^{a}, N_{3}^{a}\right\} \\
& C_{a g g} \quad\left(N_{1}^{a}, N_{2}^{a}, N_{3}^{a}\right)=\min \left(R_{1}, N_{1}^{a}\right)\left(c_{1}+f_{r}\left(r_{1}\right)\right)+\min \left(\max \left(N_{1}^{a}-R_{1}, 0\right), \max \left(N_{2}^{a}-R_{2}, 0\right)\right)\left(c_{2}+c_{\text {exc }}+f_{r}\left(r_{2}\right)\right) \\
& \quad+\quad \max \left(N_{1}^{a}-R_{1}-\max \left(R_{2}-N_{2}^{a}, 0\right), 0\right)\left(c_{3}+c_{\text {exc }}\right)+\min \left(R_{2}, N_{2}^{a}\right)\left(c_{2}+f_{r}\left(r_{2}\right)\right)+\max \left(N_{2}^{a}-R_{2}, 0\right)\left(c_{3}+c_{\text {exc }}\right)+N_{3}^{a} c_{3}
\end{aligned}
$$

with $a \in\left\{{ }^{\prime} N E^{\prime},{ }^{\prime} H^{\prime}\right\}$, respectively.

\subsection{Analytical comparison with the game model $\Gamma_{2}(N)$}

The analytical comparison of the two decision-making models, as realized for the priority heuristic through Theorem 1, is not straightforward. Hence, we present numerical results for the distribution of the drivers and the resulting aggregate cost at the $\mathrm{NE}$ of the $\Gamma_{2}(N)$ game (equation (20) and Tables 3 and 4 in Appendix) and when their decisions are driven by the lexicographic (LEX and DEBA) heuristics (equations (8)-(18) and (20)). We also plot the cost (label OPT in the respective legends) induced by a benchmark centralized reservation scheme with complete information about the parking capacity, which seeks to minimize the aggregate cost without looking into the preferences of individual drivers. Such a scheme would direct exactly $R_{1}$ drivers to on-street spots, exactly $R_{2}$ to the distant parking lot, and the remaining ones to the expensive parking lot. Our assumptions for the distributions $F_{t h r_{f}}()$, and $F_{t h r_{d}}()$ are summarized in Table 6 (the reasoning is similar to that for $F_{t h r_{c}}$, see section 4.2. 
Figure 6 plots four scenarios that are representative of the results' patterns over a much broader scenario space. Roughly speaking, scenario (a) "favors" the on-street parking choice assigning relatively high fees to the two parking lots, whereas scenario (d) serves the opposite purpose by increasing the on-street parking fee and the cruising cost, thus shrinking its fee-related advantage over parking lots. Scenarios (b) and (c) promote the cheaper parking lot (it lies closer to the trip destination and asks for a smaller fee) and the more expensive one (small fee, the cheap lot alternative is quite distant), respectively. Four main remarks are worth making when looking at these plots.

Firstly, drivers who select the on-street parking option as a result of fully rational strategic decision-making, always exceed in number the available parking spots (ref. Proposition 1). During (very) high parking demand, the excess demand ratio equals $N_{13} / R_{1}$. Likewise, excess parking demand is also induced at the $\Gamma_{2}(N)$ equilibria states for the distant parking lot; the respective ratio stabilizes to $N_{23} / R_{2}$ for high demand.

Secondly, under the same full rationality assumption, the expensive parking lot starts attracting drivers (if at all) only at high parking demand values, far exceeding the aggregate parking capacity of the other two parking resources, $R_{1}+R_{2}$. The exact value at which this happens does not only depend on how the fee of the expensive lot compares with the other two but also the capacities of the parking resources (Fig. 6b vs. 6r).

Thirdly, when drivers make choices activating the lexicographic heuristics, the distribution of drivers across the three options exhibits less variety across the four scenarios. The expensive parking lot is consistently a more competitive option, sometimes emerging as the most popular option (Fig. 6d). Over-competition is still evidenced for on-street parking capacity but not for the distant parking lot. The latter is preferred by few users in all scenarios, who do not exhaust its capacity but only at high parking demand. The exception is the second "favorable" scenario, where the cheap lot is the choice of preference for a higher portion of drivers.

Finally, and as a result of the three aforementioned trends, the aggregate cost paid by drivers for seizing a parking spot is almost always smaller when drivers activate lexicographic heuristics than when their choices are assumed to be fully rational and strategic. Namely, the $\Gamma_{2}(N)$ game equilibria predictions turn out to be consistently more pessimistic except for a few instances of low demand (scenaria (b) and (d), for $N<50$ ). In many cases, the aggregate cost under lexicographic heuristics lies very close to the minimum one that would result from the benchmark centralized 

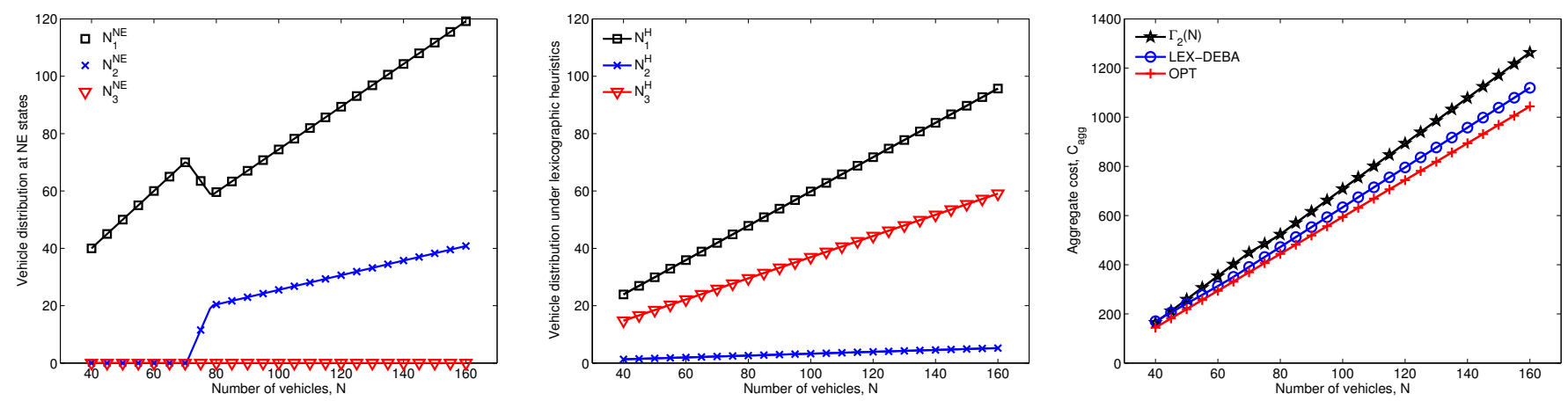

(a) $\left\{c_{1}, c_{2}, c_{3}\right\}=\{1,5,7.5\}, c_{e x c}=1,\left\{R_{1}, R_{2}\right\}=\{20,20\}, r_{2}=10$
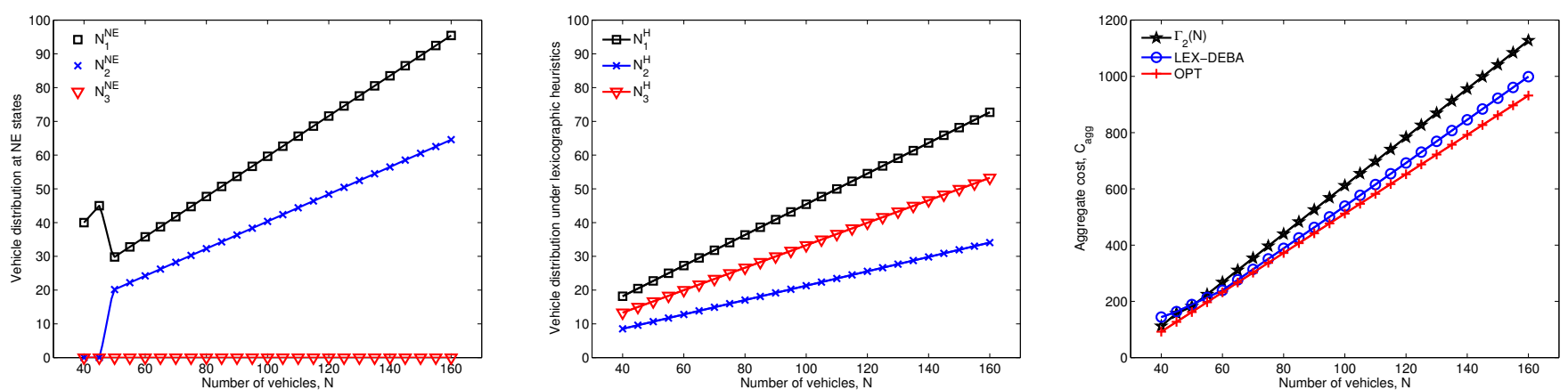

(b) $\left\{c_{1}, c_{2}, c_{3}\right\}=\{1,3,7\}, c_{e x c}=1,\left\{R_{1}, R_{2}\right\}=\{20,20\}, r_{2}=4$
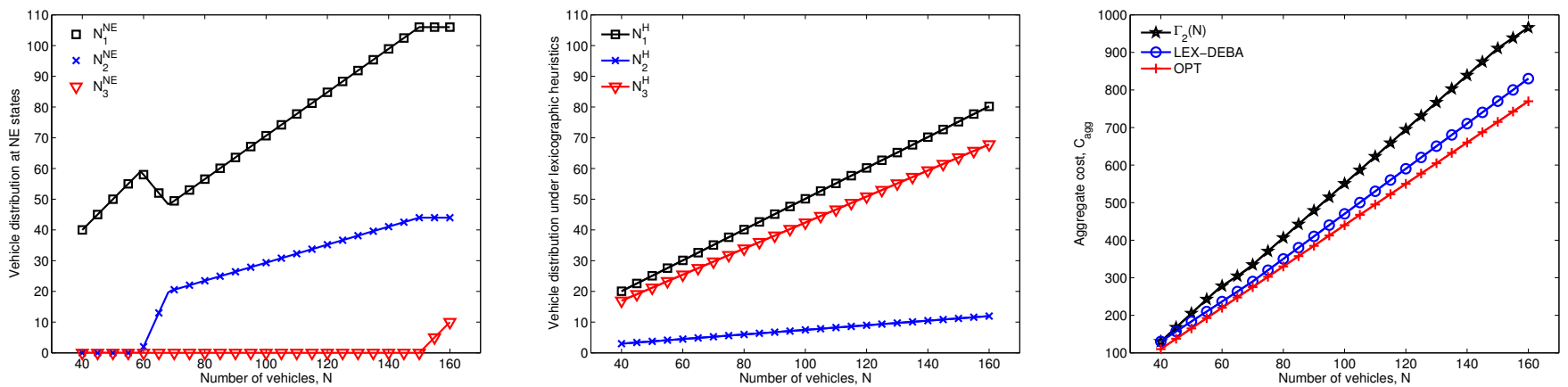

(c) $\left\{c_{1}, c_{2}, c_{3}\right\}=\{1,3.5,5.5\}, c_{e x c}=1,\left\{R_{1}, R_{2}\right\}=\{20,20\} r_{2}=8$
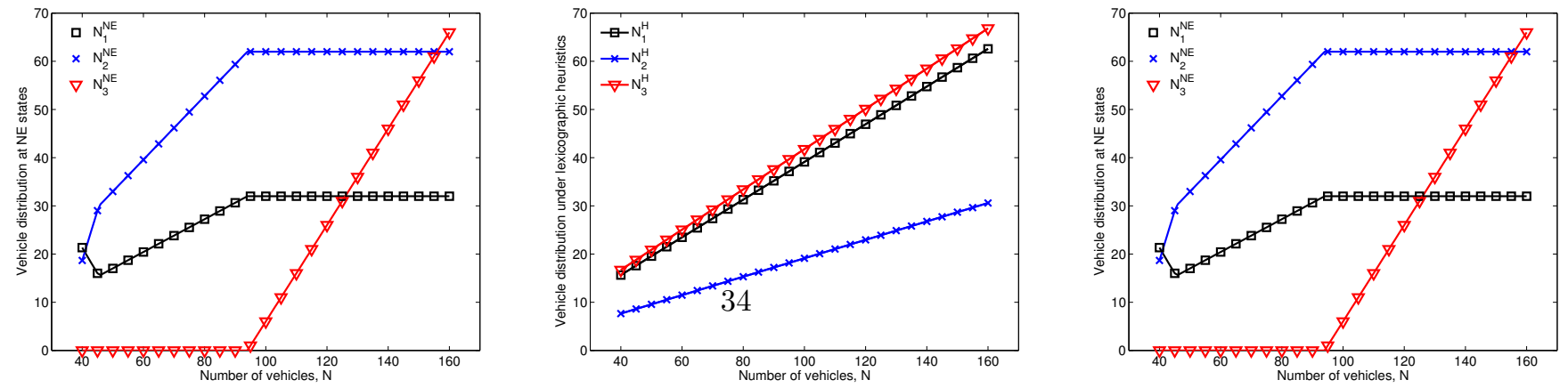

(d) $\left\{c_{1}, c_{2}, c_{3}\right\}=\{1.5,3,5\}, c_{e x c}=1.5,\left\{R_{1}, R_{2}\right\}=\{10,30\}, r_{2}=4$

Figure 6: Distribution of vehicles to the three parking resources at the $\mathrm{NE}$ of the $\Gamma_{2}(N)$ game (left) and according to the lexicographic heuristics (middle); aggregate cost under the two decision-making modes and the optimal centralized scheme (right): $r_{1}=2, r_{\max }=12, c_{\max }=8$. 
reservation scheme.

\section{Implications and open questions}

We discuss in this section the practical implications of our work as well as open questions that call for further investigation.

The analysis and results in sections 4 [6 most clearly demonstrate that our predictions about the choices drivers make and the resulting system efficacy may vary significantly depending on the adopted modeling assumptions about the way drivers decide and act; namely, whether they strategically and fully rationally analyze the decision setting looking for optimal decisions or rely on simple heuristics to reach decisions that satisfice them. However, the results as such are less instructive as to which modeling assumption is the right one in this case.

One way to respond to this question is axiomatically. For more than half a century now (e.g., Simon (1955)), it is being considered far more plausible that humans activate (lexicographic) heuristics to make choices instead of imitating players in strategic games. There is compelling evidence, for example in Ford et al. (1989) and in Katsikopoulos \& Gigerenzer (2008), that lexicographic heuristics are invoked by humans in a wide range of very different selection tasks and can justify several well-reported empirical phenomena that result from their choices. Nevertheless, the ultimate validation of the heuristics' relevance to the parking search setting demands large-scale experimentation campaigns in real or semi-real (i.e., emulator) conditions. Such experimentation is currently an open challenge for various research communities including psychologists, computer scientists, and transportation engineers. Probably the most promising path to respond to this challenge is presented by the recent generation of mobile parking application 5 that seek to change the way parking search is carried out all over the world. Running on smartphones and having the potential to collect data of unprecedented size and detail about the parking preferences and habits of their users-drivers, these applications attract strong interest as live research testbeds as well.

In particular, data from such applications would allow tackle the issue of individual preferences, which has been rather overlooked in the research agenda of cognitive decision-making heuristics. The data could be used to directly learn which heuristics users activate and how are they parameterized

\footnotetext{
${ }^{5}$ Examples include Parkmobile (http://us.parkmobile.com/), ParkMe (https://www.parkme.com), ParkAround (https://www.parkaround.com/, ParkTag (http://parktag.mobi/)
} 
for each one of them. Hence, in the case of the priority heuristic, those data would let us infer the value of the thresholds $t h r_{c}$ and $t h r_{p}$ for each user; for the LEX heuristic, how each user orders the \{parking resource type, fee, distance $\}$ decision attributes; and, for DEBA, the order of attributes plus the relevant satisfying thresholds for each attribute. The statistical modeling of drivers' heterogeneity through $F_{t h r_{f}}(x)$ and $F_{t h r_{p}}(x)$, for the priority heuristic in Section 4 , and the matching of drivers' profiles to the questionnaire data for the LEX and DEBA heuristic in Section 675 6. are simple ad-hoc ways to cater for driver heterogeneity in the absence of real "model training" data from a parking search application.

The second way to argue about the appropriateness of the two modeling assumptions is $a$ posteriori, looking at the results and assessing their plausibility. The two models differentiate in the way they prescribe the impact of the fees charged by the parking resources, but also that of their location and capacity. Figures 7 and 8 imply that the cognitive heuristic decision-making models may be much more realistic with respect to the sensitivity of the drivers' decisions to parameters such as, in this case, the difference in fees charged by the parking resources. In Fig. 7 , for example, the (higher) variations of $N_{1}$ (the expected number of drivers who decide to compete for on-street parking) with the difference $c_{2}-c_{1}$ under the priority heuristic model (dashed lines) are more in line with intuition than the $\Gamma_{1}(N)$ game predictions (solid lines); the latter are more often than not invariable to the fee difference. Likewise, in the case of two parking lots, the heuristic decision-making model in Fig. 8 generates more realistic distributions of drivers to the three parking resources. Even under a unit cost difference in the fees charged by the two lots (Fig. 8f-top row), the NE of the $\Gamma_{2}(N)$ game suggest that none of the drivers will choose the more expensive but destination-adjacent one. At the same time, as the fee $c_{3}$ increases, more and more drivers change their decision to compete for on-street parking spots in favor of driving toward the distant parking lot. On the contrary, the lexicographic heuristic model more plausibly predicts that the number of drivers choosing the expensive parking lot gracefully decreases and shifts to the other two options.

Our work has clear hints for the design of the software agents that assist drivers with the parking search task. Such agents will most probably form integral parts of broader-scope applications that will be running on devices mounted onboard the vehicles and also assist drivers with road navigation and parking manoeuvring. It will be their task to collect information about the available parking resources, the fees they charge and estimates of the parking demand from the parking assistance system infrastructure. They will then process this information to make a recommendation to the 

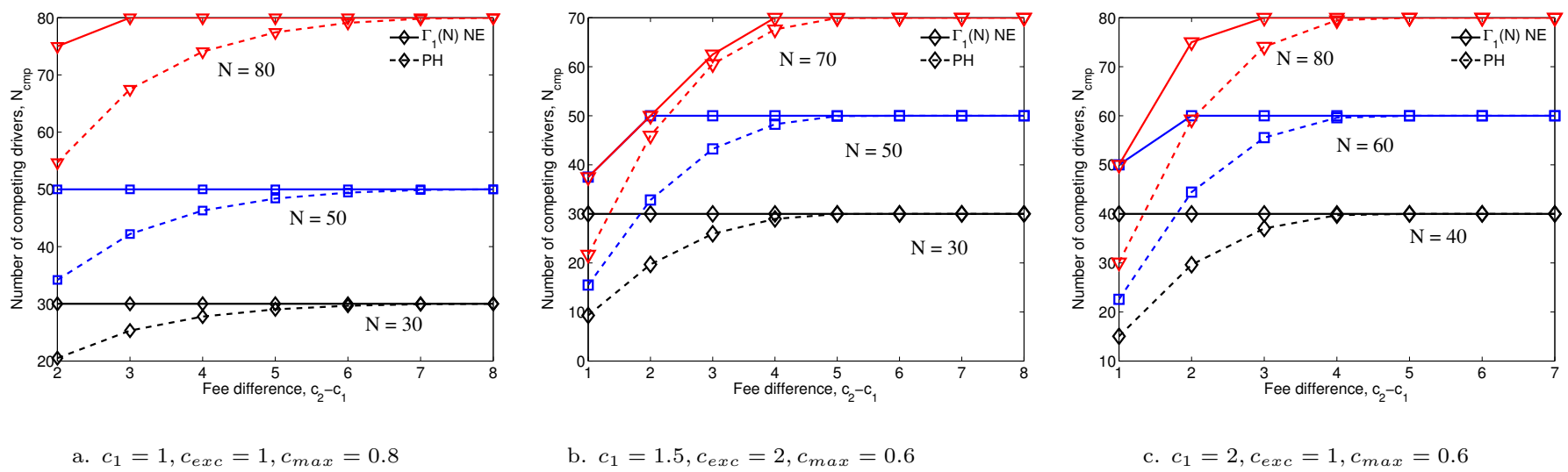

a. $c_{1}=1, c_{e x c}=1, c_{\max }=0.8$

b. $c_{1}=1.5, c_{e x c}=2, c_{\max }=0.6$

c. $c_{1}=2, c_{e x c}=1, c_{\max }=0.6$

Figure 7: Sensitivity of competing vehicles to the difference of parking resource fees: priority heuristic vs. game $\Gamma(N), R=25$.

driver as to whether to search for on-street parking space or drive to a parking lot.

One possibility to derive such recommendations is by imitating the fully rational strategic agent model: approach the drivers' collective decision setting as a game, compute the NE of this game, and make the respective recommendation to the driver. A simpler alternative would be to have the driver pass information about her preferences to the agent, possibly upon its first use by her, and then let the agent apply cognitive decision-making heuristics such as those in Sections 4 and 5 Such profile information would include, for example, whether she prefers parking lots over on-street spots, what is the acceptable fee difference between the two and how far from her destination she is willing to park. The application's mission would then become to make recommendations that respect its owner's preferences. Notably, according to the results of the paper, in the majority of the scenarios, this second option will result in a socially more efficient parking selection process.

It is less clear, and an interesting point for further investigation, how could a centralized parking management system that would positively know the drivers' profiles (which heuristics they use, how they rank the decision criteria) actively adapt and coordinate its recommendations to nudge drivers towards socially favorable choices. Such a task would involve manipulating the information that is announced towards drivers in personalized ways such as presenting them with an appropriately chosen subset of their actual alternatives. 

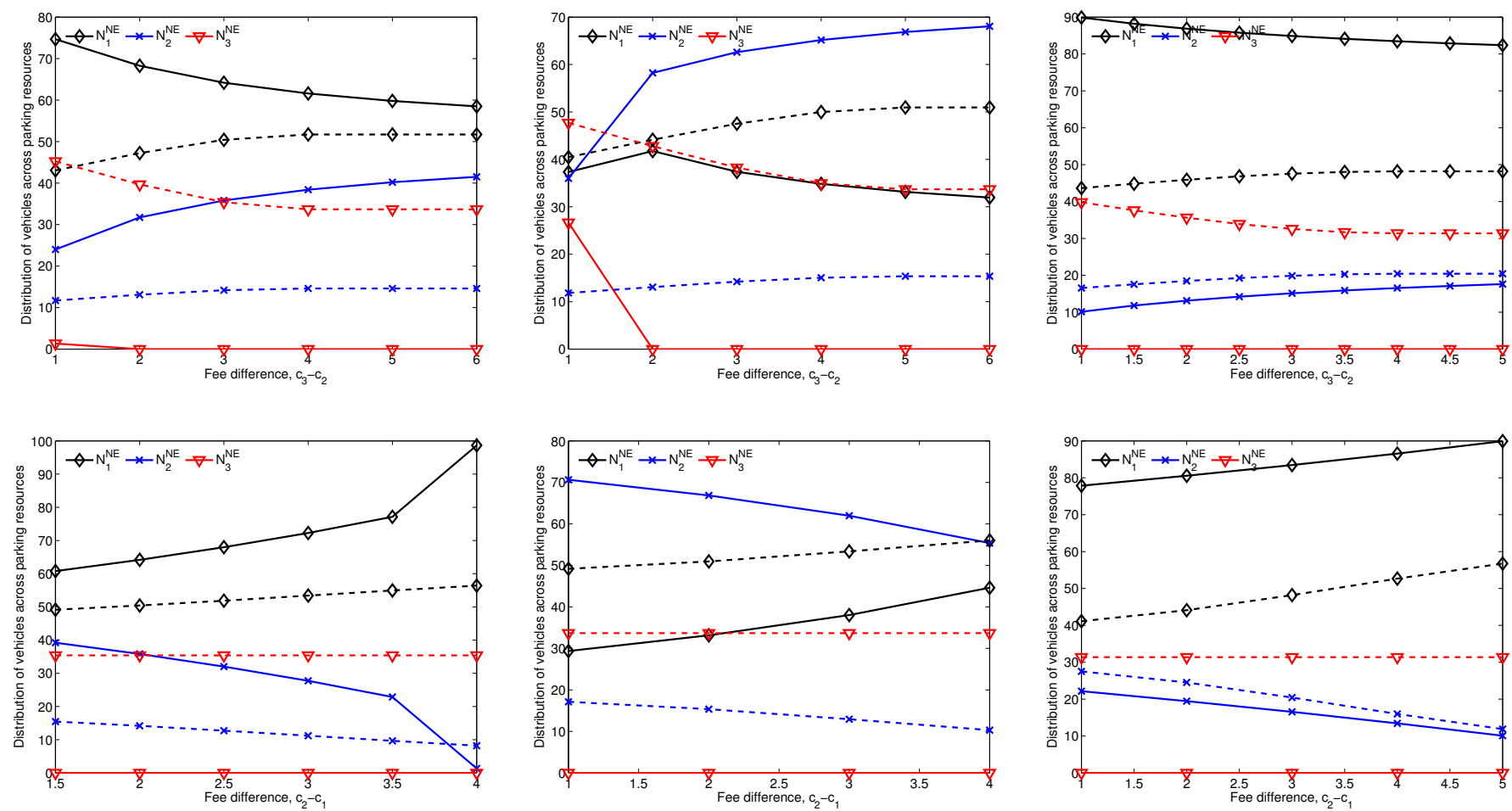

a. $R_{1}=20, R_{2}=20, c_{1}=2, r_{2}=6$

b. $R_{1}=10, R_{2}=30, c_{1}=1, r_{2}=6$

c. $R_{1}=30, R_{2}=10, c_{1}=1, r_{2}=3$

Figure 8: Sensitivity of competing vehicles to the difference of the fees charged by the two parking lots: lexicographic heuristics (dashed lines) against game $\Gamma_{2}(N)$ (solid lines), $c_{e x c}=1, r_{1}=2, r_{\max }=12, c_{\max }=8$. Top: $c_{2}=4$, bottom: $c_{3}=8$.

\section{Conclusions}

We have carried out a performance analysis study that acknowledges the possible role of cognitive heuristics in drivers' decision-making and assesses their impact on the efficiency of the parking search process. These heuristics trace their origins in the area of cognitive psychology and have seen enormous research over the last twenty years. Besides addressing the cognitive mechanics underlying human decisions, they cater naturally for humans' bounded rationality and present a radical departure from the normative model of the strategic fully rational decision-maker, who systematically seeks to maximize (minimize) its gains (losses).

In the paper, we have studied three members of the broader family of lexicographic heuristics, 
i.e., the priority, LEX, and DEBA heuristics. To this end, we have approached two typical scenarios of parking search as instances of multi-attribute choice problems with two and three alternatives, respectively. Through analysis and experimentation, we have shown that, over a broad range of scenarios' parameterizations, the parking demand is distributed more efficiently to the parking resources if we assume that drivers decide in line with these heuristics rather than strategically as fully rational agents. Finally, we have discussed the practical implications of our results for the design of parking assistance applications and outlined possible research directions.

\section{Appendix A. Proof of Theorem 1}

As already mentioned in Section 3.1, whenever $N>R$, the number of competing drivers induced

by the strategic game exceeds the on-street parking capacity, i.e., $N_{c m p}^{N E}>R$. On the contrary, under the priority heuristic, this number may generally be greater or smaller than $R$. We consider these two cases separately.

$N_{c m p}^{P H}<R$ : This implies that

$$
r_{d c}<\left(1+\frac{c_{2}}{c_{e x c}}\right) \cdot F_{t h r_{c}}^{-1}(R / N)
$$

and the difference in the aggregate cost induced by the two decision-making models is

$$
\begin{aligned}
\Delta C_{a g g} & =C_{a g g}^{P H}-C_{a g g}^{N E} \\
& =\left(R-N_{c m p}^{P H}\right)\left(c_{2}-c_{1}\right)-\left(N_{c m p}^{N E}-R\right) c_{e x c}
\end{aligned}
$$

If $N_{c m p}^{N E}=N_{0}<N$, i.e., $r_{d c}<N / R-1$, then

$$
\Delta C_{a g g}=-N_{c m p}^{P H}\left(c_{2}-c_{1}\right)<0
$$

without additional conditions.

On the other hand, if $N_{c m p}^{N E}=N$, i.e.,

$$
\begin{gathered}
r_{d c} \geq N / R-1 \\
\Delta C_{a g g}=R\left(c_{2}-c_{1}+c_{e x c}\right)-N\left(F_{t h r_{c}}\left(\frac{c_{2}-c_{1}}{c_{2}+c_{e x c}}\right)\left(c_{2}-c_{1}\right)+c_{e x c}\right)
\end{gathered}
$$

is negative when $r_{d c}<(N-R) /\left(N \cdot F_{t h r_{c}}\left(\frac{c_{2}-c_{1}}{c_{2}+c_{e x c}}\right)-R\right)$ so that, in combination with (A.4), $r_{d c}$ must satisfy

$$
\frac{N-R}{R} \leq r_{d c}<\frac{N-R}{N \cdot F_{t h r_{c}}\left(\frac{c_{2}-c_{1}}{c_{2}+c_{e x c}}\right)-R}
$$


This interval of values is non-empty only when

$$
r_{d c}<F_{t h r_{c}}^{-1}\left(\frac{2 R}{N}\right)\left(1+\frac{c_{2}}{c_{e x c}}\right)
$$

740

Constraint A.6 is inactive since, for $N_{c m p}^{P H}<R$, it is dominated by constraint (A.1).

$N_{c m p}^{P H} \geq R$ : Now $r_{d c} \geq\left(1+\frac{c_{2}}{c_{e x c}}\right) \cdot F_{t h r_{c}}^{-1}(R / N)$ and the aggregate cost under both decision-making models can be written as function of $N_{c m p}$ as

$$
C_{a g g}=R \cdot\left(c_{1}-c_{2}-c_{e x c}\right)+N \cdot c_{2}+N_{c m p} \cdot c_{e x c}
$$

so that

$$
\Delta C_{a g g}=\left(N_{c m p}^{P H}-N_{c m p}^{N E}\right) c_{e x c}
$$

and it suffices to compare the numbers of drivers that end up competing for on-street parking space under the two models.

When $N_{c m p}^{N E}=N$, i.e., $r_{d c}>N / R-1$

$$
\Delta C_{a g g}=N \cdot\left(F_{t h r_{c}}\left(\frac{r_{d c}}{1+c_{2} / c_{e x c}}\right)-1\right)<0
$$

without additional conditions. Otherwise, when $r_{d c} \leq N / R-1$, it must also hold

$$
r_{d c}>\frac{N \cdot F_{t h r_{c}}\left(\frac{r_{d c}}{1+c_{2} / c_{e x c}}\right)}{R}-1
$$

Combining (A.1)-(A.10) we get Table 2

\section{Appendix B. Proof of Theorem 2}

We provide a sketch of the proof avoiding notation formalities that burden its comprehensibility.

case: $N_{12} \leq R_{1}+R_{2}$

As long as the drivers are fewer than the on-street parking spots $R_{1}$, the NE prescribes that all of them should direct thereto. The same holds for all $N$ values up to $N_{12}$, where the expected cost of choosing to search for curbside parking reaches that of the second choice (head directly for the distant parking lot).

$$
\frac{R_{1}}{N_{12}}\left(c_{1}+f_{r}\left(r_{1}\right)\right)+\left(1-\frac{R_{1}}{N_{12}}\right)\left(c_{2}+f_{r}\left(r_{2}\right)+c_{\text {exc }}\right)=c_{2}+f_{r}\left(r_{2}\right)
$$

The individual cost drivers pay at the equilibrium remains unchanged, i.e., $c_{2}+f_{r}\left(r_{2}\right)$, as the number of drivers increases beyond $N_{12}$ and up to $R_{1}+R_{2}$, the equilibrium choice being "head for the distant parking lot" for any driver beyond the first $N_{12}$ ones. 
As soon as the drivers exceed the number of spots in the two resources, $R_{1}+R_{2}$, those who choose to compete for on-street parking should also account for the probability that they may end up in the expensive lot if neither the curbside nor the distant parking lot can serve them. On the contrary, those choosing the direct parking lot keep on paying $c_{2}+f_{r}\left(r_{2}\right)$ as long as they are fewer than $R_{2}$. The cost individual drivers pay at the equilibrium remains the same, but with the number of drivers $N_{1}^{N E}$ dropping as a function of $N$. The condition for cost indifference between the two options at the equilibrium $\left(n_{1}, n_{2}\right)$ now becomes:

$$
\frac{R_{1}}{n_{1}}\left(c_{1}+f_{r}\left(r_{1}\right)\right)+\left(1-\frac{R_{1}}{n_{1}}\right)\left[\frac{R_{2}-n_{2}}{n_{1}-R_{1}}\left(c_{2}+f_{r}\left(r_{2}\right)+c_{e x c}\right)+\left(1-\frac{R_{2}-n_{2}}{n_{1}-R_{1}}\right) \cdot\left(c_{3}+c_{e x c}\right)\right]=c_{2}+f_{r}\left(r_{2}\right)
$$

When $n_{2}=R_{2}, n_{1}=N_{13} / N_{23} R_{2}$. Hence, the system of equations consisting of (B.5) and equality $n_{1}+n_{2}=N$, describe the NE equilibria as $N$ ranges in $\left[R_{1}+R_{2}, \frac{N_{123}}{N_{23}} R_{2}\right]$.

When $N$ exceeds $\frac{N_{123}}{N_{23}} R_{2}$, the drivers are faced with a non-zero probability to end up in the expensive lot, even if they originally select the distant parking lot. The NE states $\left(n_{1}, n_{2}\right)$ should now satisfy

$$
w_{1}\left(n_{1}\right) \doteq \frac{R_{1}}{n_{1}}\left(c_{1}+f_{r}\left(r_{1}\right)\right)+\left(1-\frac{R_{1}}{n_{1}}\right)\left(c_{3}+c_{e x c}\right)=\frac{R_{2}}{n_{2}}\left(c_{2}+f_{r}\left(r_{2}\right)\right)+\left(1-\frac{R_{2}}{n_{2}}\right)\left(c_{3}+c_{e x c}\right) \doteq w_{2}\left(n_{2}\right)
$$

Since $n_{1}+n_{2}=N$, it comes out that $n_{1}=\frac{N_{13}}{N_{13}+N_{23}} N$ and $n_{2}=\frac{N_{23}}{N_{13}+N_{23}} N$. NE states with $n_{3}>0$ emerge only when the expected costs of the first and the second choice equal the cost (fees) of the expensive parking lot. This occurs for $n_{1}=N_{13}$ and $n_{2}=N_{23}$ values satisfying

$$
\begin{gathered}
\frac{R_{1}}{N_{13}}\left(c_{1}+f_{r}\left(r_{1}\right)\right)+\left(1-\frac{R_{1}}{N_{13}}\right)\left(c_{3}+c_{e x c}\right)=c_{3}, \text { and } \\
\frac{R_{2}}{N_{23}}\left(c_{2}+f_{r}\left(r_{2}\right)\right)+\left(1-\frac{R_{2}}{N_{23}}\right)\left(c_{3}+c_{e x c}\right)=c_{3}
\end{gathered}
$$

respectively. At the NE, any number of drivers beyond $N_{13}+N_{23}$ select the expensive parking lot so that the individual driver's cost is steady at $c_{3}$. Table 3 provides the full set of NE that emerge considering explicitly whether the aforementioned equilibria values are integers or not. Note that, depending on the latter and the range of $N$, multiple types of equilibrium states (each one with multiple realizations) exist. Their validity check is straightforward.

case: $N_{12}>R_{1}+R_{2}$

In this case, the equilibrium action for all drivers in $\left[0, R_{1}+R_{2}\right]$ is to compete for on-street parking; namely, the expected cost of this choice remains lower than $c_{2}+f_{r}\left(r_{2}\right)$ despite the risk of failing to seize a spot and having to cruise before heading for the distant lot. This holds also for $N>R_{1}+R_{2}$, where there is a non-zero probability that some of them will eventually have to 
park at the expensive parking lot, and up to a value $n_{1}^{\prime}$ that equalizes the expected costs of the two alternatives, i.e., on-street parking and distant parking lot:

$$
\frac{R_{1}}{n_{1}^{\prime}}\left(c_{1}+f_{r}\left(r_{1}\right)\right)+\left(1-\frac{R_{1}}{n_{1}^{\prime}}\right)\left[\frac{R_{2}}{n_{1}^{\prime}-R_{1}}\left(c_{2}+f_{r}\left(r_{2}\right)+c_{e x c}\right)+\left(1-\frac{R_{2}}{n_{1}^{\prime}-R_{1}}\right) \cdot\left(c_{3}+c_{e x c}\right)\right]=c_{2}+f_{r}\left(r_{2}\right)
$$

which yields $n_{1}^{\prime}=\frac{N_{123}-R_{2}}{N_{23}} R_{2}$. For $N>n_{1}^{\prime}$, the choice of the distant parking lot becomes competitive and the equilibrium demands a reduction of the drivers who compete for on-street parking. The choice indifference condition for $\left(n_{1}, n_{2}\right)$ at the equilibria is now:

$$
\frac{R_{1}}{n_{1}}\left(c_{1}+f_{r}\left(r_{1}\right)\right)+\left(1-\frac{R_{1}}{n_{1}}\right)\left[\frac{R_{2}-n_{2}}{n_{1}-R_{1}}\left(c_{2}+f_{r}\left(r_{2}\right)+c_{e x c}\right)+\left(1-\frac{R_{2}-n_{2}}{n_{1}-R_{1}}\right) \cdot\left(c_{3}+c_{e x c}\right)\right]=c_{2}+f_{r}\left(r_{2}\right)
$$

a) When $R_{1}<N<N_{12}, N_{1}^{N E}=N>R_{1}$.

b) For $N$ in $\left(N_{12}, R_{1}+R_{2}\right]$, the respective number is

$$
N_{1}^{N E}=N_{12}=\left(1+\frac{\left(c_{2}-c_{1}\right)+\left(f_{r}\left(r_{2}\right)-f_{r}\left(r_{1}\right)\right)}{c_{\text {exc }}}\right) R_{1}>R_{1}
$$

c) As $N$ varies in $\left(R_{1}+R_{2}, \frac{N_{123}}{N_{23}} R_{2}\right]$, it is decreasing and gets its lowest value $N_{1}^{N E}=R_{2} \frac{N_{13}}{N_{23}}$ when $N=\frac{N_{123}}{N_{23}} R_{2}$. It is straightforward to show that

$$
R_{2} \frac{N_{13}}{N_{23}}=\frac{c_{3}+c_{e x c}-c_{1}-f_{r}\left(r_{1}\right)}{c_{3}+c_{e x c}-c_{2}-f_{r}\left(r_{2}\right)} R_{1}>\frac{c_{3}+c_{e x c}-c_{2}-f_{r}\left(r_{2}\right)}{c_{3}+c_{e x c}-c_{2}-f_{r}\left(r_{2}\right)} R_{1}=R_{1}
$$


d) As $N$ increases from $\frac{N_{123}}{N_{23}} R_{2}$ towards $N_{123}$, the value of $N_{1}^{N E}$ grows from its lowest value in (C.1) towards higher values. Therefore,

$$
N_{1}^{N E} \geq R_{2} \frac{N_{13}}{N_{23}}>R_{1} \quad \forall N \in\left(\frac{N_{123}}{N_{23}} R_{2}, N_{123}\right]
$$

e) Finally, for $N>N_{13}+N_{23}$ the equilibrium value is

$$
N_{1}^{N E}=N_{13}=\left(1+\frac{c_{3}-c_{1}-f_{r}\left(r_{1}\right)}{c_{e x c}}\right) R_{1}>R_{1}
$$

Note that over-competition is also induced for the distant parking lot, albeit at demand values $N>\frac{N_{123}}{N_{23}} R_{2}$.

case: $N_{12}>R_{1}+R_{2}$

The proof for the case $N_{12}>R_{1}+R_{2}$ does not need any additional effort with respect to its counterpart for $N_{12} \leq R_{1}+R_{2}$. Comparing Table 4 with Table 3 shows that the minimum values obtained by the number of drivers selecting the on-street parking option at equilibrium, $N_{1}^{N E}$, are the same (in Table 3 there is an additional interval for $N$, throughout which $N_{1}^{N E}=N_{12}$ ). Therefore, the over-competition effect is consistently evidenced for all values of $N>R$, also when $N_{12}>R_{1}+R_{2}$.

\section{References}

R. Arnott (2006). 'Spatial competition between parking garages and downtown parking policy'. Transport Policy (Elsevier) pp. 458-469.

D. Ayala, et al. (2011). 'Parking Slot Assignment Games'. In Proc. 19th ACM SIGSPATIAL GIS.

S. D. Boyles, et al. (2015). 'Parking search equilibrium on a network'. Transportation Research Part B: Methodological 81, Part 2:390-409.

E. Brandstatter, et al. (2006). 'The priority heuristic: making choices without trade-offs'. Psychological Review 113(2):409-432.

M. Caliskan, et al. (2006). 'Decentralized discovery of free parking places'. In Proc. 3rd VANET (in conjunction with ACM MobiCom), Los Angeles, CA, USA.

J. Cao \& M. Menendez (2015). 'System dynamics of urban traffic based on its parking-related-states'. Transportation Research Part B: Methodological 81, Part 3:718 - 736.

Z. Chen, et al. (2016). 'Analysis of Advanced Management of Curbside Parking'. Transportation Research Record: Journal of the Transportation Research Board 2567:57-66. 
X. Di \& H. X. Liu (2016). 'Boundedly rational route choice behavior: A review of models and methodologies'. Transportation Research Part B: Methodological 85:142 - 179.

L. Du \& S. Gong (2016). 'Stochastic Poisson game for an online decentralized and coordinated parking mechanism'. Transportation Research Part B: Methodological 87:44-63.

M. Florian \& M. Los (1980). 'Impact of the supply of parking spaces on parking lot choice'. Transportation Research Part B: Methodological 14(1):155 - 163.

J. K. Ford, et al. (1989). 'Process tracing methods: Contributions, problems and neglected research questions'. Organizational Behavior and Human Decision Processes pp. 42, 75-117.

D. Gale \& L. Shapley (1962). 'College admissions and the stability of marriage'. The American Mathematical Monthly 69(1):9 - 15 .

G. Gigerenzer \& D. G. Goldstein (1996). 'Reasoning the fast and frugal way: Models of bounded rationality'. Psychological Review 103:650-669.

G. Gigerenzer, et al. (2011). Heuristics: The Foundations of Adaptive Behavior. New York: Oxford University Press.

G. Gigerenzer \& R. Selten (2001). Bounded rationality: the adaptive toolbox. MIT Press, Cambridge, MA, USA.

S. R. Greenberg (2015). 'Using Innovation and Technology to Improve City Services'. IBM Center for the The Business of Government, White paper under Using Technologies Series .

F. He, et al. (2015). 'Pricing of parking games with atomic players'. Transportation Research Part B: Methodological 73:1-12.

A. Hester, et al. (2002). 'Drivers' Parking Decisions: Advanced Parking Management Systems'. Journal of Transportation Engineering 128(1).

R. M. Hogarth \& N. Karelaia (2005). 'Simple models for multiattribute choice with many alternatives: When it does and does not pay to face tradeoffs with binary attributes?'. Management Science 51(12):1860-1872.

J. Hunt \& S. Teply (1993). 'A nested logit model of parking location choice'. Transportation Research Part B: Methodological 27(4):253-265.

J. Hutchinson, et al. (2012). 'Car parking as a game between simple heuristics'. In P. M. Todd, G. Gigerenzer and the ABC research group, Ecological rationality: Intelligence in the world pp. 454-484.

D. Kahneman (2011). Thinking, Fast and Slow. Farrar, Straus and Giroux.

M. Karaliopoulos, et al. (2014). 'Bounded Rationality Can Increase Parking Search Efficiency'. In Pro- 
ceedings of the 15th ACM International Symposium on Mobile Ad Hoc Networking and Computing, MobiHoc '14, pp. 195-204, Philadelphia, Pennsylvania, USA. ACM.

K. V. Katsikopoulos (2011). 'Psychological heuristics for making inferences: Definition, performance, and the emerging theory and practice'. Decision Analysis 8(1):10-29.

K. V. Katsikopoulos (2014). 'Bounded rationality: The two cultures'. Journal of Economic Methodology 21(4):361-374.

K. V. Katsikopoulos, et al. (2002). 'Risk attitude reversals in drivers' route choice when range of travel time information is provided'. Human Factors: The Journal of the Human Factors and Ergonomics Society 44(3):466-473.

K. V. Katsikopoulos \& G. Gigerenzer (2008). 'One-reason decision-making: Modeling violations of expected utility theory'. Journal of Risk and Uncertainty 37(1):35-56.

E. Kokolaki, et al. (2013). 'Leveraging Information in Parking Assistance Systems'. Vehicular Technology, IEEE Transactions on 62(9):4309-4317.

E. Koutsoupias \& C. H. Papadimitriou (2009). 'Worst-case Equilibria'. Computer Science Review 3(2):6569.

E. Kurz-Milcke \& G. Gigerenzer (2007). 'Heuristic decision making'. Marketing : Journal of research and management $\mathbf{3}(1)$ :48-56.

W. H. Lam, et al. (2006). 'Modeling time-dependent travel choice problems in road networks with multiple user classes and multiple parking facilities'. Transportation Research Part B: Methodological 40(5):368 $-395$.

T. A. Lambe (1996). 'Driver choice of parking in the city'. Socio-Economic Planning Sciences 30(3):207 219.

C. Lei \& Y. Ouyang (2017). 'Dynamic pricing and reservation for intelligent urban parking management'. Transportation Research Part C: Emerging Technologies 77:226 - 244.

J. MacQueen \& J. Miller (1960). 'Optimal Persistence Policies'. Operations Research 8(3):362-380.

J. Marewski \& G. Gigerenzer (2012). 'Heuristic decision making in medicine'. Dialogues in Clinical Neuroscience 14(1):77-89.

J. W. Payne, et al. (1993). The adaptive decision maker. New York: Cambridge University Press.

Z. S. Qian \& R. Rajagopal (2014). 'Optimal occupancy-driven parking pricing under demand uncertainties and traveler heterogeneity: A stochastic control approach'. Transportation Research Part B: Methodological 67:144 - 165 . 
Z. S. Qian, et al. (2012). 'Managing morning commute traffic with parking'. Transportation Research Part B: Methodological 46(7):894 - 916 .

A. Rubinstein (1998). Modeling bounded rationality. MIT Press, Cambridge, MA, USA.

D. Shoup (2006). 'Cruising for parking'. Transport Policy 13(3).

D. C. Shoup (1999). 'The trouble with minimum parking requirements'. Elsevier Transportation Research Part A: Policy and Practice 33(7):549-574.

H. A. Simon (1955). 'A Behavioral Model of Rational Choice'. The Quarterly Journal of Economics 69(1):99-118.

H. A. Simon (1986). 'Rationality in Psychology and Economics'. The Journal of Business 59(4):S209-S224.

D. Van Der Goot (1982). 'A model to describe the choice of parking places'. Transportation Research Part A: General 16(2):109 - 115.

A. Zanella, et al. (2014). 'Internet of Things for Smart Cities'. IEEE Internet of Things Journal 1(1):22-32. 